\title{
Estrategias de género en negociación salarial: Evidencia experimental ${ }^{*}$
}

\author{
Catrihel Greppi \\ Octubre, 2016 \\ Maestría en Economía \\ Universidad Nacional de La Plata \\ Director \\ Guillermo Cruces
}

\begin{abstract}
Resumen
La evidencia observacional sobre brechas de género en el mercado laboral es abundante. Sin embargo, existe mucho menos consenso sobre los mecanismos causales detrás de estas diferencias en resultados laborales de hombres y mujeres. Mientras que parte de la evidencia apunta a la existencia de discriminación por parte de los empleadores (e.g. el lado de la demanda), otra parte, en cambio, se enfoca en cuestiones de comportamiento y preferencias de los trabajadores (e.g. el lado de la oferta). En este trabajo, nos preguntamos si los trabajadores internalizan el género del empleador en sus decisiones y, en base a esto, ajustan sus estrategias de competencia y negociación. Con esta pregunta en mente, realizamos un experimento de campo en una plataforma de trabajo en línea. Encontramos que tanto hombres como mujeres ofrecen realizar los mismos trabajos por un salario hasta un $22 \%$ menor si el empleador es hombre, en comparación con una empleadora mujer o de género neutro. Proponemos una posible interpretación de estos resultados a través de un modelo de estereotipos en el cual los trabajadores compiten por precio ante un empleador hombre y por calidad ante una empleadora mujer. Contribuimos al debate basado en evidencia sobre este tema desde una perspectiva poco explorada hasta ahora y en un contexto laboral emergente.
\end{abstract}

\footnotetext{
*Esta tesis de maestría forma parte de un proyecto de investigación más amplio en el que trabajamos en conjunto con Hernán Galperin (USC), Guillermo Cruces (CEDLAS-UNLP-CONICET) y María Fernanda Viecens (UdeSACONICET). El experimento que realizamos obtuvo aprobación de un comité independiente de ética (IRB Review). Agradecemos a la gente de Nubelo por la asistencia técnica durante la investigación (en particular, a Paz Madina, Nicolás Araujo y Marcos Sánchez), a Ricardo Pérez-Truglia y Martin Rossi por comentarios iniciales al proyecto y a los participantes del seminario de avances de tesis de la Maestría en Economía UNLP por sus sugerencias (en particular, a Mariana Marchionni, Natalia Porto, Leopoldo Tornarolli, Joaquín Serrano y Joaquín Coleff). Todos los errores u omisiones son responsabilidad de la autora. Personalmente, agradezco a Guillermo, Hernán y Fernanda por enseñarme, guiarme, y permitirme formar parte de una experiencia integral de investigación desde el principio y a mis compañeros y amigos del CEDLAS por el aprendizaje y apoyo que me brindaron en esta etapa.
} 


\section{Introducción}

A pesar de que la evidencia observacional sobre brechas de género en el mercado laboral es abundante (Goldin, 2006, Gasparini y Marchionni, 2015), existe menor consenso y entendimiento sobre los mecanismos subyancentes que resultan en menores ingresos y carreras truncadas para las mujeres. Parte de la evidencia apunta a discriminación en las decisiones de contratación y ascensos por el lado de la demanda de trabajo, mientras que una literatura más reciente enfatiza cuestiones de comportamiento y preferencias, tales como la auto-selección y las diferencias en atributos psicológicos y de identidad de grupo en el lado de la oferta. Sin embargo, poco se sabe acerca del efecto del género del empleador sobre los resultados laborales. En particular, cabe preguntarse si los trabajadores internalizan el género del empleador y ajustan sus estrategias de competencia y negociación acordemente.

La literatura previa encuentra que las mujeres no solo tienen mayores probabilidades de establecerse en firmas con menores rentas o beneficios potenciales sino que son, además, menos propensas a negociar sus salarios y condiciones laborales. En contraste, la literatura enfocada en el impacto de la escasez de información y ambiguedad sobre las decisiones y, en particular, Leibbrandt y List (2015) sostienen que las mujeres prefieren ambientes de trabajo en los cuales las reglas de determinación de los salarios son explícitas y concretas. En estos casos, señalan los autores, las mujeres negocian tanto como los hombres.

Este trabajo va más allá y sostiene que este comportamiento no es independiente del lado de la demanda de trabajo. En este sentido, nos concentramos en una dimensión del contexto laboral que no ha sido lo suficientemente explorada hasta ahora: el impacto del género del empleador sobre la voluntad del trabajador de aceptar un trabajo y negociar por un mayor salario.

Las plataformas de trabajo en línea brindan la oportunidad de examinar estas preguntas de manera costo-efectiva. No solo permiten testear hipótesis sobre las interacciones de género en procesos de contratación y negociación -y comenzar a comprender los mecanismos que subyacen a los desbalances registrados en ingresos y trayectorias laborales entre hombres y mujeres- sino que, además, brindan información valiosa como contexto laboral real y emergente. En este trabajo, recurrimos a la economía experimental para identificar diferencias en el comportamiento de género en estos mercados laborales. En particular, exploramos los diferenciales en participación en ingresos asociados con las interacciones trabajador-empleador en diferentes contextos de negociación salarial. Más específicamente, nos preguntamos si los candidatos -hombres y mujeres- que compiten por trabajos en una plataforma online ajustan, por un lado, sus estrategias de búsqueda y negociación y, por el otro, sus precios de reserva en base al género inferido del empleador. La relevancia de esta pregunta radica en queel comportamiento en las etapas iniciales de las relaciones laborales en negociación y competencia podrían traducirse en diferencias en resultados económicos.

Para explorar estos interrogantes, diseñamos un experimento de campo en el cual invitamos a una muestra de freelancers en una plataforma a postularse a seis ofertas de trabajos idénticas excepto por dos factores: el género del empleador y las posibilidades de negociación salarial. Los freelancers que aceptan la invitación a postular deben enviar una propuesta con su remuneración pretendida. En este sentido, exploramos dos niveles del contrato laboral. En una primera etapa, nos interesa la decisión de los freelancers de postular a un trabajo en el que un empleador de un cierto tipo propone negociación o la proscribe explícitamente y, en una segunda etapa, la decisión de negociar por un mayor salario una vez que se decide postular al trabajo. Los freelancers no saben que forman parte de un experimento, pero se realizan contrataciones efectivas para cada uno de los trabajos publicados. En este sentido, el experimento está diseñado de tal forma que simula 
transacciones reales que suceden a diario en este tipo de mercados. Tanto en el contexto en el que se propone un salario negociable como en aquel en el que se ofrece un salario no negociable, los freelancers tienen la posibilidad de competir por precio, tiempo de entrega y mediante diferentes señales de calidad, lo cual permite explorar, además, diferencias en las estrategias de postulación ante ofertas de empleadores de distinto género.

Posteamos seis (6) ofertas de trabajo en la plataforma en línea: una para cada género del empleador (hombre, mujer o neutral) en dos contextos de negociación diferentes (precio explícitamente negociable versus precio explícitamente no negociable). Este diseño implica que, potencialmente, contamos con doce (12) comparaciones de interés, si tenemos en cuenta el género de los trabajadores. No obstante, solo seis de estas comparaciones son causales en el sentido de que el parámetro de interés es manipulado exógenamente. Cada una de estas ofertas de trabajo es privada y solo los freelancers que invitamos a postular e indicar su remuneración pretendida pueden acceder a ellas. En total, aleatorizamos estas seis ofertas en una muestra de 2800 freelancers españoles activos en la plataforma en el año 2015. De estas 2800 invitaciones enviadas, recibimos alrededor de 500 propuestas: es decir, una tasa de respuesta del $18 \%$.

No encontramos diferencias en preferencias por contextos de negociación (o tasa de respuesta entre anuncios) entre los freelancers asignados a cada uno de nuestros empleadores hombre y mujer, aunque los freelancers tienen una mayor probabilidad de aceptar una invitación a un trabajo si empleador es neutro o no revela el género, en particular, en contextos donde el precio del trabajo no es negociable. Por otra parte, los freelancers hombres tienen mayor probabilidad de incurrir en competencia por precio incluso cuando la negociación no está permitida o cuando el precio del trabajo es explícito. Es decir, los hombres desafían la regla de precio fijo y ofrecen realizar el trabajo por un menor salario que el ofrecido, siempre que el empleador sea hombre. Este resultado es consistente con la literatura que sostiene que los hombres se desempeñan mejor que las mujeres en ambientes donde las posibilidades de negociación son ambiguas. En tales contextos, según Leibbrandt y List (2015), los hombres se llevan una cantidad desproporcionada del excedente porque compiten por precio o negocian más frecuentemente.

En cuanto a la segunda dimensión de nuestro experimento, encontramos que el género del empleador inferido por los candidatos potenciales influye sobre la distribución del excedente entre nuestros empleadores. El empleador neutro en género nos ayuda a identificar hasta que punto estas diferencias se deben, simplemente, al hecho de revelar el género de un empleador. En efecto, argumentamos que existe un claro "efecto empleador hombre" sobre las remuneraciones pretendidas por los freelancers, dado que nuestros empleadores mujer y neutro son virtualmente equivalentes. En concreto, tanto trabajadores hombres como mujeres se ofrecen a realizar los mismos trabajos por menores salarios si el empleador es de género masculino. La diferencia se traduce en un salario potencial un $22 \%$ menor, lo cual puede interpretarse también como un premio monetario de un $22 \%$ para el empleador hombre.

Una posible interpretación de estos resultados consiste en la existencia de estereotipos de género por parte de los trabajadores. Las diferencias salariales podrían deberse a la existencia de productos o trabajos diferenciados que dependan del género del empleador. Más específicamente, se propone un modelo en el cual los trabajadores compiten por calidad ante la empleadora mujer (e.g. piden un mayor salario) y por precio ante el empleador hombre (e.g. piden un menor salario).

Nuestros resultados brindan información sobre determinantes salariales en tanto proveen una intuición y cuantifican la importancia de las interacciones de género en las decisiones de participación, competencia y negociación en el mercado laboral. Realizar el experimento en una plataforma en línea nos permite testear estas teorías en un contexto donde la información tiene un gran valor 
marginal como determinante del matching y la distribución del excedente. Estas plataformas son, además, representativas de contextos de trabajo emergentes en donde las interacciones económicas son más impersonales y las características de los agentes deben ser inferidas a partir de escasa información disponible. En este sentido, nuestras estimaciones podrían representar una cota inferior (o lower bound), si esperamos que las diferencias se incrementen en las interacciones cara a cara.

El trabajo está organizado de la siguiente forma. En la siguiente sección 2, comentamos el contexto de estudio y los datos utilizados. En la sección 3, presentamos el diseño experimental en detalle. En la sección 4, reportamos los resultados experimentales. La sección 5 presenta un modelo posible para interpretar estos resultados obtenidos en un marco de estereotipos de género. La sección 6 concluye.

\section{Contexto y datos}

\subsection{Brechas salariales: ¿discriminación, comportamiento o ambos?}

Existe discriminación de género cuando hombres y mujeres igualmente productivos reciben diferentes compensaciones (Azmat y Petrongolo, 2014, Bertrand y Duflo, 2016). Gran parte de la literatura empírica sobre brechas de género en salarios y participación intenta explicar hasta qué punto se deben a la existencia de discriminación en el mercado laboral y hasta qué punto al comportamiento o preferencias de los individuos.

Incluso entre los trabajos que exploran discriminación, resulta importante distinguir, en una primera instancia, aquellos que encuentran discriminación pura (e.g. resultante de gustos o preferencias) de los que atribuyen los resultados a discriminación estadística. En ambos casos, los agentes toman decisiones en base a factores exógenos a la productividad de los trabajadores, aunque en el primero recurren a gustos o preferencias y, en el segundo, a estereotipos.

Asimismo, una vez que se establece que existe discriminación (pura o estadística), surge la necesidad de explicarla y proponer políticas para mitigar sus efectos nocivos. En el caso de discriminación estadística y estereotipos, las recomendaciones de política suelen surgir de tratamientos de información. Es decir, dado que los estereotipos se definen como atajos ante la ausencia de información relevante u otras señales sobre la productividad de los trabajadores, la crencialización o señales podrían mitigar su influencia (Goldin, 2002). La información también resulta valiosa en otros sentidos que van más allá de cuestiones de estereotipos. Cullen y Pakzad-Hurson (2014), por ejemplo, muestran que el conocimiento acerca del pago a competidores o colegas cambia sustancialmente el poder de negociación de los trabajadores.

Por otra parte, otros autores sostienen que parte de las diferencias salariales observadas se deben al comportamiento y preferencias de los individuos. Este comportamiento puede traducirse, por ejemplo, en segregación ocupacional: las mujeres se concentran en labores que pagan menos de por si o en empresas en las cuales existen menores rentas para distribuir (Goldin, 2002, Card et al, 2015) o evitan las negociaciones salariales (Leibbrandt y List, 2015). La economía experimental, por su parte, se ha concentrado en preferencias por riesgo y competencia. Estos autores argumentan, por ejemplo, que las mujeres son más aversas al riesgo y ganan menos en las negociaciones (Azmat y Petrongolo, 2014).

Otro fenómeno vinculado con el comportamiento diferencial que a menudo se cita para explicar la brecha consiste en la identidad de género: la existencia e influencia de normas sociales acerca de lo que es apropiado y no que hagan hombres y mujeres. Esta identidad tiene implicancias no solo sobre la segregación ocupacional mencionada anteriormente, sino también sobre la participación laboral, 
la asignación del trabajo dentro del hogar y la propensión a negociar y o competir (Bertrand, 2011, Bertrand et al, 2015).

Es importante notar que la existencia de segregación u otros diferenciales comportamentales no implica la ausencia de discriminación ya que ambos fenómenos podrían encontrarse fuertemente vinculados. En efecto, la discriminación podría generar cambios en los incentivos y, por ende, en el comportamiento de los individuos que resulten en las brechas observadas. Janssen et al (2014), por ejemplo, analiza la influencia de las actitudes sociales sobre la igualdad de género en las decisiones de las firmas y encuentra que las firmas en regiones en donde se acepta socialmente la desigualdad de género pagan salarios más discriminatorios. Goldin (2002), por su parte, argumenta que es justamente la discriminación de género la que genera segregación en el mercado laboral: la contratación de mujeres disminuye el prestigio de tareas convencionalmente masculinas.

Mientras que gran parte de la literatura de discriminación se ha concentrado en las decisiones de los empleadores acerca de la remuneración y/o contratación de trabajadores pertenecientes a distintos grupos minoritarios (por ejemplo, las mujeres), en este trabajo focalizamos en la otra dimensión del mercado laboral: el lado de la oferta de trabajo. Es decir, nos interesa, a diferencia de la mayoría de los trabajos citados anteriormente, identificar el impacto del género del empleador sobre el comportamiento de los trabajadores en términos de negociación y competencia e intentar establecer hasta qué punto las diferencias encontradas se explican por discriminación pura (e.g. gustos o preferencias de los trabajadores) o discriminación estadística (e.g. estereotipos de género aplicados al empleador).

\subsection{Experimentos en economía laboral}

Probar la existencia de discriminación (tanto pura como estadística) es todo un desafío empírico, dado que la productividad y otros atributos, en general, no son observables de manera directa (Bertrand y Duflo, 2016). Es por ello que cada vez más autores recurren a la economía experimental. El objetivo de esta rama consiste en generar variación exógena en variables de interés para ser capaces de identificar relaciones causales y explicar mecanismos subyacentes. Partiendo de una base de la teoría o incluso preconcepciones, se busca testear hipótesis sobre el funcionamiento de contextos de interacción económica entre agentes (List y Rasul, 2011). Típicamente, el procedimiento consiste en asignar aleatoriamente un grupo a un tratamiento o intervención y comparar sus resultados con el grupo asignado al control (Chen et al, 2009).

La principal ventaja de estos estudios, respecto a métodos no experimentales, consiste en que se crea un pool artificial de participantes del mercado laboral que garantiza que no existirán diferencias entre grupos y, por tanto, permiten identificar una relación causal de interés (Chen et al, 2009). En términos econométricos, se podría plantear una relación:

$$
Y=\alpha+\beta G+\varepsilon
$$

En la cual $Y$ representa el resultado de interés (tasa de respuesta o salario) y $G$ una variable binaria que implica pertenencia a un determinado grupo minoritario (población afroamericana o mujeres). En un experimento controlado, el estimador de MCO $\hat{\beta}$ (o simplemente la diferencia de medias entre grupos) provee una estimación de discriminación (Neumark, 2016).

Asimismo, los estudios experimentales permiten superar limitaciones de datos dado que, a priori, no se trabaja con una base de datos existente sino que se generan observaciones. En efecto, a través 
de cálculos de potencia se puede determinar el tamaño de muestra necesario para identificar el efecto de interés y determinar la factibilidad y costo del estudio. Estos experimentos pueden realizarse tanto en laboratorios como en contextos laborales reales (o de campo).

En cuanto al primer grupo, los experimentos de laboratorio, si bien se han utilizado más ampliamente para intentar explicar interacciones de género y sus efectos sobre resultados del mercado laboral, presentan mayores limitaciones en términos de validez externa.

Algunos ejemplos incluyen estudios como el Chen et al (2015), quienes diseñan un experimento de subastas en el cual revelan el género del oponente y encuentran que las mujeres hacen ofertas monetarias más altas que los hombres, siempre que compiten contra otras mujeres. Este estudio apoya la idea acerca de la importancia de otros factores del contexto, en particular, el género de los competidores, como determinante de resultados. Heilman et al (2004), por su parte, estudian el efecto que tiene el éxito de una mujer en una tarea dominantemente masculina y encuentran que, cuando se les reconoce el éxito, se las aprueba menos y se las desestima más que a hombres comparables. Esta penalidad social, además, tiene efectos potencialmente negativos sobre sus carreras en términos de evaluaciones y recomendaciones. La idea detrás del estudio consiste en que, bajo algunas circunstancias, no resulta suficiente con probar la competitividad de las mujeres para mitigar los estereotipos.

En cuanto a los experimentos de campo, la literatura experimental en el mercado laboral que busca testear la existencia de discriminación se concentra, en gran parte, en estudios de auditoría o correspondencia ${ }^{1}$.

El diseño experimental tradicional consiste en postular candidatos ficticios a anuncios reales de empleo (vía teléfono, CV o entrevista presencial) que son virtualmente idénticos en características observables, excepto por el grupo de interés al cual pertenecen (de raza o género, por ejemplo).

En general, el foco está puesto en los resultados de contratación (en el caso de las auditorías) o tasa de respuesta (en el caso de los estudios de correspondencia) por parte del empleador (ArceoGomez y Campos-Vázquez, 2013, Newmark, 2016). Booth y Leigh (2010), por ejemplo, replican un experimento de campo de este estilo en Australia, pero para ocupaciones relativamente dominadas por mujeres. Encuentran que el sesgo positivo en contratación hacia las mujeres se incrementa con la proporción de mujeres en la ocupación.

Este tipo de experimentos de campo también se ha utilizado para testear la eficacia de políticas reparadoras, por ejemplo, las de affirmative action. Ibañez et al (2015) llevan a cabo tres experimentos en Colombia en los cuales comparan las características de los candidatos que postulan antes de que se les informe sobre la política con aquellos que postulan una vez informados. Encuentran que las ganancias superan a los costos en términos de desalentar candidatos masculinos.

Leibbrandt y List (2015) exploran la otra dimensión del proceso de contratación: la del empleador, con el objetivo de testear si las mujeres evitan las negociaciones salariales. Para ello, diseñan un experimento de campo en Estados Unidos en el cual aleatorizan potenciales candidatos a distintos trabajos, en los cuales varían el tipo de contrato: negociación salarial explícita versus negociación salarial ambigua (e.g. no mencionan la posibilidad de negociar el salario). Los autores encuentran que, en contextos ambiguos, las mujeres tienen mayor probabilidad de señalar su disposición a trabajar por un menor salario, mientras que, en los casos en los cuales la alternativa negociación es explícita, las diferencias desaparecen.

\footnotetext{
${ }^{1}$ La diferencia consiste en la forma de postular a los candidatos ficticios. En el primer caso (estudios de auditoría), la postulación es presencial mientras que, en los estudios de correspondencia, los candidatos existen en papel o electrónicamente.
} 
Nuestro diseño experimental comparte varias de las ventajas del diseño mencionado en el párrafo anterior (Leibbrandt y List, 2015). En particular, también estudiamos el comportamiento de los individuos en un contexto laboral real, sin que los participantes sepan que forman parte de un experimento o están siendo observados. Asimismo, al igual que nosotros, observan dos etapas de diferencias de género en negociación: la decisión de postular a un determinado contrato y la voluntad de negociar el salario una vez que el candidato postula, como así también su disposición a trabajar por un salario menor al ofrecido inicialmente.

Por otra parte, las principales desventajas de estos estudios, que también reconocemos, tienen que ver con la validez externa de las conclusiones obtenidas. Más especificamente, dado que el género no es un factor aleatorio, se debe recurrir a perfiles ficticios para poder aleatorizarlo e identificar su efecto causal. Por esta razón y por otras que detallamos en las secciones siguientes, resulta difícil garantizar la validez externa o extrapolar los resultados obtenidos a otros contextos de mayor escala (Chen et al, 2009), cuestión que debe tenerse presente desde las etapas de diseño experimental hasta la interpretación de los resultados.

\subsection{Plataformas de empleo en línea}

Las plataformas de empleo en línea, un contexto laboral emergente y con cada vez más presencia, funcionan como intermediarias entre trabajadores y empleadores de todo el mundo. Se especializan en contratos laborales de corto plazo, siendo algunos ejemplos oDesk, Elance o Nubelo, con la cual trabajamos en este estudio. La ventaja de Nubelo consiste, por un lado, en que es la plataforma con mayor presencia en el mercado hispanoparlante y, por el otro, contamos con acceso interno a datos sobre todas las transacciones realizadas desde el año 2012 a la fecha.

El funcionamiento de la plataforma consiste en reunir empleadores que postean trabajos (en general, de corto plazo) y trabajadores que buscan trabajos (a través de un buscador interno). En este sentido, los empleadores actúan como compradores de servicios, buscan y contratan a trabajadores independientes para que los realicen de manera remota ( $\mathrm{Ng}$ y Leung, 2015). Los freelancers (e.g. trabajadores independientes), por su parte, hacen ofertas monetarias (y cualitativas) y compiten por los trabajos disponibles en la plataforma en cada momento del tiempo.

Una propuesta de empleo consiste en un texto (con un mínimo número de palabras) y una cantidad monetaria o precio por la cual el freelancer estaría dispuesto a realizar el trabajo. El empleador recibe las propuestas y elige una (o, en contados casos, más de una) para contratar. A la hora de tomar su decisión tiene acceso, además, al historial de contrataciones del trabajador, sus valoraciones, su nombre, entre otro tipo de información que el freelancer decide revelar (tales como un CV, su foto de perfil, etc.).

Un componente clave que contribuye al buen funcionamiento es el sistema reputacional en el cual, luego de efectuarse una transacción, el empleador valora o puntua al trabajador y el trabajo realizado (otorgándole de a una a cinco estrellas) y lo recomienda a futuros empleadores mediante un texto que queda registrado en su perfil. De la misma manera, el trabajador tiene la oportunidad de valorar al empleador. La literatura muestra que las valoraciones positivas (o feedback) son sumamente importantes en estos contextos laborales en los cuales la información disponible es tan escasa que su valor marginal se incrementa exponencialmente (Pallais, 2013, Stanton y Thomas, 2014). 


\section{Diseño experimental}

\subsection{Set Up básico}

\subsubsection{Perfil de empresa}

Con el objetivo de identificar diferencias de género en estrategias de competencia y negociación de salarios, diseñamos un experimento de campo en la plataforma de trabajo en línea Nubelo.com. Para llevar a cabo el experimento, creamos, en primer lugar, un perfil ficticio de empresa y realizamos algunas contrataciones reales antes de salir a campo para obtener un historial de actividad o contrataciones efectivas y valoraciones positivas (figura 6). Este procedimiento inicial es importante porque, al igual que en cualquier situación real de contratación, necesitamos resultar atractivos y confiables para los candidatos potenciales que reciben nuestras ofertas de trabajo. En términos experimentales, este paso contribuye a maximizar nuestra tasa de respuesta (o take-up) la cual determina nuestra capacidad de identificar efectos estadísticamente (o potencia estadística). Asimismo, los pilotos realizados previamente a la intervención tuvieron como objetivo determinar si el experimento era factible y si la pregunta de investigación tenía sentido económico en este contexto (Glennerster y Takavarasha, 2013).

Los anuncios de trabajo se suben en el mismo perfil de empresa por una cuestión logística y también de validez interna: el hecho de que las invitaciones partan desde el mismo perfil evita posibles diferencias que podrían surgir en el medio de un experimento entre empleadores. A modo de ejemplo, si las invitaciones de trabajo a los freelancers partiesen desde diferentes perfiles en la plataforma en línea (e.g. un perfil para cada empleador), no podríamos garantizar que algunos perfiles recibiesen valoraciones diferentes y en diferentes momentos o etapas del experimento, lo cual invalidaría su uso en futuras etapas ya que dejarían de ser comparables (e.g. se violaría el principio de ceteris paribus).

Otro potencial inconveniente en línea con este razonamiento tiene que ver con la posibilidad de que freelancers en la plataforma observasen distintos perfiles de empleadores ofreciendo trabajos idénticos. Esto último generaría sospechas o dudas que invalidarían la validez externa e interna del experimento pejudicando, además, la tasa de respuesta. Sin embargo, esta posibilidad no es factible dado que la privacidad del perfil de empresa que utilizamos está protegida: ni el perfil de la empresa ni los trabajos publicados reciente o históricamente aparecen en búsquedas dentro o fuera de la plataforma en línea.

En concreto, lo que manipulamos no es el perfil de la empresa o empleador propiamente dicho, sino el representante de la empresa que envía las invitaciones u ofertas de trabajo en cada caso.

\subsubsection{Ofertas de trabajo}

El siguiente paso consiste en postear en el perfil de la empresa los seis anuncios de trabajo idénticos, excepto por dos factores: el género del empleador (o representante que envía la oferta de trabajo) y el contexto de negociación (figura 6c). En el primer caso, seleccionamos los nombres de

hombre (José Antonio), mujer (María Carmen) y apellido (García) más frecuentes en España, según el Instituto Nacional de Estadística del país. El hecho de que los nombres utilizados sean fácilmente identificables como femeninos o masculinos aporta a la validez externa de nuestros resultados, dado que el efecto que medimos es el del género inferido del empleador por los freelancers que participan del experimento.

En el segundo caso, definimos el tratamiento negociable como aquel en el cual el precio del trabajo que figura en el anuncio es explícitamente negociable en un rango entre 25 y 250 euros. Este 
rango se define en base a un análisis de datos observacionales en la línea de base y pilotos previos al experimento con el fin de obtener un precio razonable, teniendo en cuenta el trade-off existente con la tasa de respuesta: un rango demasiado bajo podría desalentar las candidaturas, mientras que un rango demasiado generoso podría generar sospechas acerca del empleador y el anuncio. Por otra parte, la indicación de un rango en el caso negociable tiene con objetivo brindar un precio de referencia del valor de un trabajo de ese estilo, en particular, a los freelancers que no cuentan con experiencia en estas plataformas. Asimismo, nos permite disminuir la influencia de potenciales ofertas extremas (y poco realistas) que pudiesen perjudicarnos en términos de potencia estadística. ${ }^{2}$

Por otra parte, el tratamiento no negociable es aquel en el cual el anuncio de trabajo ofrece un precio fijo de 250 euros, no negociable. Este precio se eligió con el objetivo de hacer este brazo del experimento comparable en términos de incentivos con aquel en el cual el precio es negociable: en ambos casos, los freelancers tienen la posibilidad de llevarse 250 euros. La diferencia radica en que en el tratamiento negociable deben realizar un esfuerzo extra y pedir los 250 euros y no un monto menor, disminuyendo la probabilidad esperada de ganar el trabajo.

En todos los casos, indicamos que evaluaremos todas las propuestas recibidas en una fecha predeterminada. Esta aclaración cumple con tres propósitos. En primer lugar, dejamos implícito que el freelancer invitado a postular está compitiendo con otras propuestas por el trabajo (contexto competitivo). En segundo lugar, damos suficiente tiempo a los freelancers para postular (aunque la gran mayoría postula durante los dos primeros días después de recibir la invitación), a los fines de favorecer la tasa de respuesta. Por último, específicamos que evaluaremos todas las propuestas al final del período de postulación otorgado para no desincentivar postulaciones tardías.

En total, subimos seis ofertas de trabajo o anuncios a la plataforma: una para cada empleador en dos contextos de negociación diferentes. El texto de los anuncios se presenta a continuación en la figura 1. Asimismo, con fines ilustrativos, incluimos una captura de pantalla de un anuncio en el apéndice de este trabajo (figura 6b).

Por último, el trabajo o tarea ofrecida consiste en una transcripción simple de un video de una hora, aproximadamente, sobre un tema de Marketing. Esta elección surje de un análisis de los proyectos más atrativos en la plataforma (e.g. aquellos que reciben mayor cantidad de propuestas en promedio). Se tuvo especial cuidado en elegir una tarea neutral al género que no resultase desproporcionadamente atractiva para candidatos masculinos o femeninos, como podría suceder en el caso de una traducción, y que fuese representativa del tipo de tareas que se ofrecen en la plataforma. Una tarea sesgada hacia un cierto género habría puesto en desventaja a los miembros del género opuesto, lo cual se traduciría en menos negociación o menores remuneraciones pretendidas por su parte. Asimismo, los freelancers podrían inferir la composición de género del pool de competidores interesados en obtener el empleo.

Otorgamos, además, un plazo de entrega generoso (de cuatro semanas) para favorecer la tasa de respuesta.

\footnotetext{
${ }^{2}$ Es importante tener en cuenta que 1 hora de video o audio implica entre 5 y 6 horas de trabajo de transcripción efectivo, que es en general lo que determina el precio total del trabajo. La calidad del audio también influye sobre la cotización. El valor de una hora de transcripción en español en el mercado es de 50 euros (www.voxtraducciones.com/tarifas-traduccion, por ejemplo). Datos observacionales de la plataforma indican que trabajos de transcripción en español se han valuado en 70 euros, aproximadamente. En pilotos previos, recibimos ofertas muy heterogéneas a partir de los 20 euros, siendo el valor de la máxima oferta recibida por una transcripción de este estilo de 1000 euros y la siguiente más alta de 450 euros (precio total).
} 
Figura 1: Texto de los anuncios de trabajo

Hola, [te contacto de, mi nombre es José Antonio García/María Carmen García y trabajo en] Soluciones de Marketing, una empresa especializada EN MARKETING DIGITAL. Estamos BUSCANDO UNA PERSONA PARA TRANSCRIBIR EL VÍDEO DE UNA PONENCIA (EL TEXTO LUEGO SERÁ EDITADO POR LA EMPRESA PARA PUBLICARSE EN UN BLOG). EL VIDEO TRATA SOBRE UN TEMA DE MARKETING Y TIENE UNA DURACIÓN DE 53 MINUTOS, ESTÁ EN IDIOMA ESPAÑOL Y LA CALIDAD DEL audio es muy buena. El plazo de entrega es de hasta 4 Semanas (en archivo WORD).

\section{PRECIO NEGOCIABLE}

OFRECEMOS UN PRECIO NEGOCIABLE ENTRE 25 Y 250 EUROS POR TODO EL TRABAJO. El día lunes 9 de MAYO EVALUAREMOS tOdAS las PROPUESTAS RECIBIDAS. NO SE

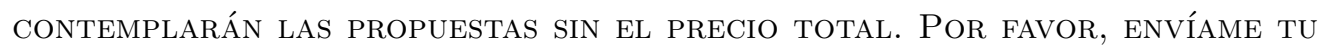
propuesta lo antes posible. Gracias.

PRECIO NO NEGOCIABLE

OfRECEMOS UN PRECIO NO NEGOCIABle de 250 EuRos POR TOdO EL TRABAJO. El día lunes 9 DE MAYO EVAluaremos todas las Propuestas RECibidas. Por FAVOR, ENVÍAME TU PROPUESTA LO ANTES POSIBLE. GRACIAS.

\subsection{Asignación al tratamiento}

\subsubsection{Muestra de freelancers}

La selección de la sub-muestra de individuos de la población sobre la cual se aleatoriza los tratamientos tiene, claramente, implicancias en términos de validez externa de cualquier experimento. Esto es así porque si bien el diseño experimental garantiza validez interna, la muestra utilizada puede no ser representativa de la población de interés y, en consecuencia, los resultados obtenidos no podrán ser extrapolables a cualquier situación o contexto ajeno al estudiado.

Nuestro pool de candidatos potenciales está formado por todos aquellos freelancers españoles activos en la plataforma en el año 2015, en las categorías de (1) Traducción y Redacción, (2) Marketing y Ventas, (3) Negocios y (4) Soporte Administrativo. Las dos primeras categorías (Traducción y Marketing) son dos de las cuatro categorías más representativas en Nubelo, siendo la tercera Diseño y Multimedia y la cuarta Desarrollo Web y Móvil. Sin embargo, estas últimas no presentan suficiente balance de género como para extraer una muestra funcional de trabajadores hombres y mujeres. Además, la tarea seleccionada (e.g. transcripción de un video sobre marketing) debería resultar de mayor interés a los freelancers en las cuatro categorías elegidas.

A pesar de que no contamos con variables representativas que indiquen con precisión cuestiones socio-demográficas del pool de candidatos utilizado en el experimento, una encuesta posttratamiento ${ }^{3}$ brinda cierta intuición de que esta tiene un nivel educativo alto, con un gran porcentaje de individuos con nivel universitario completo o incluso posgrado.

Este último punto es importante porque, en términos de validez externa, nos concentramos en una parte de la distribución que no ha sido tan estudiada en tanto brechas de género, aunque estudios previos muestran que las mujeres educadas tienen mayores probabilidades de elegir no negociar o competir, lo cual se traduce en un costo significativo para la sociedad.

\footnotetext{
${ }^{3}$ En esta encuesta que realizamos a los individuos que contestaron las ofertas de trabajo obtuvimos una tasa de respuesta del $65 \%$.
} 


\subsubsection{Aleatorización}

Como ya se mencionó en secciones anteriores, la validez interna del experimento viene dada por la asignación aleatoria a los tratamientos. Esta aleatorización garantiza que, en valor esperado, no habrán diferencias en observables e inobservables entre los grupos asignados a los distintos tratamientos, más allá de los factores que nos interesan: el contexto de negociación (precio explícitamente negociable, precio explícitamente no negociable) y el género del empleador (José, María, Neutro). Esto es así porque la asignación a los distintos tratamientos de interés es aleatoria y, en consecuencia, ortogonal a las características previas de los potenciales participantes que pudiesen afectar los resultados de interés. En este sentido, contamos con un diseño factorial $2 \times 3$ que se ilustra en la figura 2 .

Figura 2:

Asignación aleatoria al tratamiento

[1] Freelancer

\begin{tabular}{|c|c|c|c|}
\hline \multirow{4}{*}{ 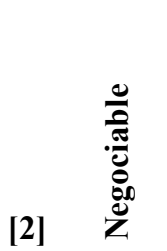 } & [3] Empleador & Hombre & Mujer \\
\hline & Hombre & HH-N & HM-N \\
\hline & Neutro & $\mathrm{NH}-\mathrm{N}$ & NM-N \\
\hline & Mujer & MH-N & MM-N \\
\hline \multirow{3}{*}{ Z } & Hombre & HH-NN & HM-NN \\
\hline & Neutro & NH-NN & NM-NN \\
\hline & Mujer & MH-NN & MM-NN \\
\hline
\end{tabular}

A diferencia de otros estudios experimentales más simples en los cuales existe un grupo control (e.g. individuos no asignados al tratamiento) y un grupo tratamiento, en este caso, como ilustra la figura 2, estamos interesados en las comparaciones de a pares: es decir, el efecto de ser asignado al tratamiento $T_{i}$ (recibe oferta de empleo de empleador hombre) con respecto al efecto de ser asignado al tratamiento $T_{j}$ (recibe oferta de empleo de empleadora mujer o de empleador neutro).

El efecto que nos interesa medir, por un lado, es el efecto de haber sido invitado a postular a la oferta de trabajo por el empleador de género g sobre la tasa de respuesta (e.g. la decisión individual de postular o no postular) y la remuneración pretendida. Dado que los freelancers postulan a través de un monto (acompañado de un texto u otros elementos que apoyan la candidatura), la decisión de postular está vinculada con el envío del precio esperado por el trabajo (tal cual se solicita en el anuncio descripto en la sección anterior).

El supuesto de identificación detrás de este procedimiento consiste en que, en promedio, el resultado para los freelancers asignados al tratamiento $T_{1}$ habría sido el mismo que el de los del grupo $T_{2}$ si, en cambio, hubiesen sido asignados a este último tratamiento. Esto nos permite identificar el efecto causal del género del empleador sobre los resultados, aislando dicho efecto de potenciales sesgos de selección que existirían en situaciones no experimentales.

El proceso de aleatorización involucra tanto muestreo aleatorio como asignación aleatoria al tratamiento. En efecto en una primera etapa (1), seleccionamos una muestra aleatoria de freelancers 
de la población definida en la sección anterior (es decir, españoles en la plataforma de Nubelo en un dado período) y estratificamos por género de los trabajadores o freelancers, es decir, dividimos la muestra de individuos en hombres y mujeres. Al seleccionar una submuestra de esta forma garantizamos que los individuos que participen del experimento serán representativos de la población de interés (Glennerster y Takavarasha, 2013). Asimismo, la estratificación es importante para garantizar el balance en términos de género, dado que la aleatorización lo garantiza solo en valor esperado y es un factor sumamente relevante en términos de potenciales interacciones con la variable de interés.

La segunda etapa (2) de la aleatorización consiste en, dentro de cada muestra resultante de trabajadores (e.g. hombres y mujeres), asignar los freelancers al tratamiento u oferta de trabajo de precio negociable o no negociable.

Por último, en la tercera etapa (3), en cada grupo que resulta de las dos etapas anteriores, asignamos los freelancers al emplador hombre (José), mujer (María) o neutro.

Es importante notar que, más allá de la asignación aleatoria a las diferentes ofertas de trabajo, dado que los freelancers asignados al tratamiento $T_{i}$ eligen si responder a la invitación o no, en el caso en que se busca identificar el efecto sobre monto o salario, existe la posibilidad de selección por participación: los individuos que responden al anuncio del empleador hombre, por ejemplo, pueden no ser comparables con los individuos que responden al anuncio de la empleadora mujer. Este problema se exacerba cuanto menor sea la tasa de respuesta o take-up del experimento.

A modo de ejemplo, supongamos que el grupo de freelancers que postula a la empleadora de género femenino tiene mayor educación o experiencia, en promedio. En este caso, una diferencia positiva en remuneración pretendida entre empleadores se debería, simplemente, a un efecto composición. Es decir, los individuos que postulen a los distintos empleadores serían diferentes en otros factores no controlados por el experimento. Aún en este caso, estaríamos frente a un efecto género del empleador, aunque indirecto (a través de los incentivos a postular al trabajo para distintos grupos de freelancers). Por otra parte, la existencia y dirección de este efecto composición puede testearse ex post si se cuenta con covariables pre-tratamiento relevantes o si identificamos diferencias significativas en tasa de respuesta a los diferentes empleadores.

En suma, este diseño controlado nos permite atribuir las diferencias que resultan (de comparaciones de a pares) en tasa de respuesta y monto o salario al género del empleador inferido por los freelancers y al contexto de negociación.

Por último, si bien la asignación aleatoria a los distintos tratamientos garantiza balance en características previas en valor esperado, los grupos serán estadísticamente idénticos siempre que contemos con una muestra lo suficientemente grande. En nuestro caso, al trabajar con una muestra chica, a priori, es posible que existan algunas diferencias significativas en las covariables de los grupos. En la tabla 4 se presenta el balance en características pre-tratamiento de la muestra asignada a cada empleador y contexto de negociación en algunas covariables disponibles en la base de línea y potencialmente correlacionadas con el resultado de interés, como ser: el momento del día en que se le envío la propuesta (tiempo), el valor promedio de propuestas que el freelancer hizo en la plataforma (variable de resultado pre-tratamiento), el valor promedio de proyectos a los que postuló, el total de propuestas enviadas por el freelancer desde que ingresó a la plataforma (actividad) y la fecha de registro del freelancer (antiguedad).

\subsubsection{Invitaciones}

En total, enviamos 2.800 invitaciones y recibimos alrededor de 500 propuestas, lo cual se traduce en una tasa de respuesta del $18 \%$. 
Para realizar el envío de las invitaciones explotamos una característica de la plataforma que permite invitaciones privadas de empleadores a potenciales candidatos pre-seleccionados a través del buscador (en nuestro caso, de una base de datos de freelancers activos en 2015). Esto implica que solo los freelancers invitados por nosotros tienen acceso al anuncio de trabajo que reciben y que un freelacer asignado al tratamiento $x$ no sabe de la existencia del tratamiento $y$.

Solo interactuamos con los freelancers (más allá del envío de las invitaciones) de manera posterior a que observamos los resultados de interés (respuesta y remuneración pretendida) para, por ejemplo, solicitarles que llenen un formulario o responder preguntas. Esto incrementa la validez externa de nuestros resultados dado que, cuidadosamente, replicamos las condiciones bajo las cuales tienen lugar las transacciones diariamente en este tipo de mercados.

El tiempo es un determinate clave del éxito de estas transacciones y, en particular, de los resultados que observamos (e.g. contratación y monto) en estos mercados laborales. Es de esperar diferencias entre freelancers que reciben una invitación u oferta laboral en diferentes momentos del día o, incluso, en diferentes días de la semana. Es por ello que en nuestro experimento alternamos el envío de las invitaciones de manera tal que no existan diferencias significativas y sistemáticas entre empleadores o tratamientos en esta variable, y completamos el envío total en el transcurso de un día y medio. Por una cuestión de precisión, en algunas especificaciones incluimos el orden del envío de la invitación como control.

Por otra parte, para determinar el momento (día y horario) óptimo de envío de las invitaciones (y garantizarnos una tasa de respuesta representativa) analizamos datos observacionales sobre el tránsito en la plataforma en España. Estos datos indican que la mayor proporción de propuestas se envían los días lunes, martes y miércoles a primeras horas de la mañana.

En todos los casos, al finalizar el experimento, seleccionamos una propuesta entre todas las recibidas y realizamos una contratación real, de la misma forma en que se desarrollan las transacciones diarias en la plataforma.

Por último, es importante notar que, aunque la competencia queda implícita en el anuncio, los freelancers no pueden observar ni la cantidad ni el monto de las otras propuestas recibidas por el empleador. Esto es importante dado que, de poder hacerlo, las propuestas tardías estarían claramente sesgadas por las primeras ofertas recibidas.

\section{Resultados experimentales}

\subsection{Discusión preliminar}

\subsubsection{Precio negociable versus precio no negociable}

Como se mencionó en la sección 3, la primera dimensión de nuestro experimento consiste en asignar freelancers (hombres y mujeres) a uno de dos posibles escenarios de negociación salarial. En el contexto donde el precio del trabajo es no negociable, invitamos a los trabajadores a postular a un trabajo valuado en 250 euros y explicitamente informamos que se trata de un valor fijo. En el contexto de negociación, en cambio, invitamos a otro grupo de freelancers a enviar sus propuestas para exactamente el mismo trabajo, con la diferencia de que, en este caso, brindamos la alternativa de negociar el valor del trabajo dentro de un cierto rango (entre 25 y 250 euros).

En términos de resultados, nos interesa tanto la decisión de postular o no al trabajo como la remuneración esperada. Nuestra medida de negociación (o competencia por precio) consiste en recibir una propuesta diferente (por encima o por debajo) al valor máximo que el empleador ofrece 
pagar por el trabajo, que es siempre el mismo (250). Esta especificación nos permite observar la proporción de la renta disponible que el freelancer pide al empleador.

Definimos el excedente potencial del empleador como la diferencia proporcional entre su precio de reserva anunciado (e.g. cota superior) y la propuesta promedio que recibe: ${ }^{4}$

$$
\begin{gathered}
E=(W-w) / W \\
W=250 \\
w \epsilon[25,250]
\end{gathered}
$$

Dado que los 250 euros están disponibles en ambos escenarios, las diferencias en monto o respuesta entre estas dos alternativas debería reflejar la voluntad de los freelancers de realizar un "esfuerzo extra" en el contexto de negociación para obtener el salario potencial máximo. Sin embargo, las posibles interpretaciones no se limitan únicamente a preferencias por negociación. Si bien únicamente aquellos freelancers que reciben nuestra invitación pueden ver el anuncio de trabajo y subir ofertas, nuestro anuncio deja implícito que están compitiendo con otros freelancers por el contrato.

En particular, en el contexto de negociación, los freelancers tienen la oportunidad de llevarse 250 euros; si deciden postular pero piden menos dinero, lo cual sucede en la mayoría de los casos, no solo están señalando su voluntad de negociar, sino también su voluntad de competir por precio para obtener el trabajo, conscientes de que su propuesta podría ser rechazada por el empleador. En términos de las expectativas del freelancer, este rechazo podría resultar en una contratación (e.g. el empleador contrata a alguien más) o no (el empleador decide cancelar la oferta de trabajo y no continuar con el proceso). En cualquier caso, una vez que el freelancer decide que le conviene postular, intenta maximizar la probabilidad de ser contratado o ganar el trabajo, la cual es una función decreciente del precio que pide por este.

Asimismo, mientras que en el contexto de negociación los freelancers pueden -y son incentivados a- competir por precio (es decir, aumentar sus probabilidades de obtener el trabajo al pedir un salario por debajo de la cota superior indicada), los medios de competencia son menos claros en el contexto en el cual el precio del trabajo es no negociable. No obstante, aún en este caso, las posibilidades de competir existen y deben ser tenidas en cuenta. A modo de ejemplo, el sistema de postulación de la platafoma requiere que los freelancers brinden una determinada cantidad de información no monetaria a los empleadores en sus propuestas, en forma de un texto y una mínima cantidad de palabras, mediante la cual se presentan de manera escrita.

En estos textos, los freelancers revelan información voluntaria (en el sentido de que eligen que incluir o exculir) al empleador acerca de su experiencia laboral previa (en la plataforma o en otros contextos laborales), credenciales y/o las razones por las cuales creen que el empleador debería elegir su perfil por encima de los de los demás competidores. Esto implica que pueden competir de distintas maneras (monetarias y no monetarias): ofertando un precio debajo del precio de reserva revelado por el empleador, ofreciendo entregar el trabajo antes del plazo propuesto, o por calidad potencial del trabajo. Los freelancers podrían esperar que el empleador pondere más la experiencia y las credenciales en casos en los cuales la competencia por precio no es la opción más evidente.

Es importante notar que nada impide que los freelancers pidan un mayor (o menor) salario en el contexto en el cual el precio es explícitamente no negociable. Un comportamiento de este tipo implicaría desafiar las reglas establecidas por el empleador. En efecto, elvalor máximo de las

\footnotetext{
${ }^{4}$ Se indica en las estimaciones cuando se conservan las propuestas fuera de este rango anunciado.
} 
propuestas recibidas es el doble del máximo precio potencial ofrecido (e.g. 500 euros). Sin embargo, este escenario es poco probable en los datos. Es decir, en general, los freelancers se abstienen de hacer una oferta antes que pedir un salario mayor a los 250 euros no negociables. No obstante, es mucho más frecuente el caso en el cual los freelancers ofrecen realizar el trabajo por un menor precio que el ofrecido.

Del mismo modo, en el contexto de precio negociable, los freelancers podrían ofertar por debajo de la cota inferior (e.g. 25 euros), por encima de la cota superior (e.g. 250 euros) o algún valor intermedio. Menores precios señalan competencia por precio mientras que valores superiores se interpretan como negociación. El valor de la oferta máxima recibida por el trabajo fue cuatro veces la cota superior indicada (e.g. 1000 euros), mientras que la mínima oferta fue menos de la mitad de la cota inferior (e.g. 10 euros). Esta negociación o competencia por fuera del rango es más probable en el contexto explícitamente negociable, aunque incluso en este caso es poco probable.

\subsubsection{Género del empleador}

El otro brazo de nuestro experimento consiste en asignar género -o no- al empleador detrás de cada uno de estos contextos de negociación. Es decir, dentro de cada uno de estos escenarios (precio negociable y precio no negociable), invitamos -aleatoriamente- a hombres y mujeres a postular a trabajos idénticos excepto por el género del empleador.

Manipulamos el género del empleador a través de nombres fácilmente identificables. Más específicamente, las ofertas de trabajo provienen de un representante de la misma companía (Soluciones de Marketing) que se presenta como José Antonio García, María Carmen García - los dos nombres más frecuentes para hombres y mujeres en España, respectivamente. En el caso del empleador de género neutro, no revelamos ningún nombre: el empleador se presenta simplemente como un representante de la companía.

\subsubsection{Especificación}

En suma, nos enfocamos en dos tipos de resultado: tasa de respuesta y monto (e.g. remuneración pretendida) para los 12 grupos de interés: freelancers hombres y mujeres asignados a ofertas de empleo de empleador hombre, mujer y neutro en contextos con y sin negociación salarial. Esto implica que contamos con 6 comparaciones de a pares para cada uno de nuestros resultados (figura 2).

Otra ventaja de nuestro diseño experimental es que nos permite separar resultados para candidatos hombres y mujeres: el foco no está en comparar resultados de los trabajadores hombres relativos a las trabajadoras mujeres, sino en comparar preferencias entre distintos contextos de negociación dentro de cada género de freelancers (por ejemplo, hombres asignados a negociación versus hombres asignados a precio fijo). Asimismo, aunque lo que asignamos aleatoriamente es el género del

empleador, podemos también extraer conclusiones interesantes al comparar el comportamiento (o brechas por género del empleador) para ambos tipos de freelancers (hombres y mujeres).

Nuestros resultados principales pueden, entonces, resumirse la siguiente ecuación:

$$
Y_{i g b}=\alpha+\beta_{1}(J)+\beta_{2}(M)+\beta_{3}(N)+\beta_{4}(N * J)+\beta_{4}(N * M)+\theta X+\epsilon
$$

Donde $Y_{i g b}$ representa el resultado de interés (respuesta binaria o monto) del individuo $i$ asignado al empleador de género $g$ en el contexto de negociación $b, J$ es un indicador de empleador hombre 
(José), $M$ es un indicador de empleadora mujer (María), $N$ indica el contexto de negociación, $X$ es un vector de covariables pre-tratamiento que se incluyen en algunas especificaciones para incrementar la precisión de los estimadores (tales como el orden de envío de las propuestas) y $\epsilon$ es un término de error. Las regresiones se corren para el grupo de freelancers hombres y mujeres por separado, a menos que se indique lo contrario.

Presentamos un resumen desagregado de estos resultados en las tablas 1 y 2 , regresiones con controles pre-tratamiento en la tabla $3 y$ en las secciones siguientes discutimos los resultados.

\subsection{Participación}

Como ya mencionamos en secciones anteriores, la tasa de respuesta agregada del experimento es del 18\%. La tabla 1 muestra las diferencias en tasa de respuesta a invitaciones enviadas -o postulaciones- entre los tratamientos de precio negociable y precio no negociable, distinguiendo los resultados, además, por género del empleador y género del freelancer.

No encontramos diferencias significativas en tasa de respuesta (o preferencias) por contextos de negociación para freelancers hombres ni mujeres, lo cual podría deberse a falta de potencia estadística, dado que algunos coeficientes son importantes en magnitud (tabla IC). En particular, este resultado no contradice lo encontrado por la literatura, aunque tampoco lo ratifica. Leibbrandt and List (2012), por ejemplo, encuentra que los hombres, en comparación con las mujeres, prefieren ambientes de trabajo en los cuales las reglas de determinación salarial son ambiguas, lo cual sería equivalente a nuestro contexto de negociación.

Se encuentran, en cambio, algunas dinámicas interesantes en cuanto al género del empleador (tabla IB). Dentro del contexto de negociación, por ejemplo, los trabajadores (hombres y mujeres) tienen menos probabilidad de postular al trabajo si la invitación proviene del empleador hombre, con respecto al empleador de género neutro. La diferencia es estadísticamente significativa y de -4 puntos porcentuales, o de casi un $20 \%$ con respecto con la media del grupo base.

Por otra parte, en el contexto en el cual el salario no es negociable, encontramos una brecha similar entre los empleadores hombre y mujer respecto al empleador que no revela el género. Los freelancers tienen menor probabilidad de postular si el género del empleador es tanto masculino como femenino, con respecto al empleador de género neutro, aunque la significatividad y magnitud de este efecto se debe a las trabajadoras mujeres. La magnitud de la diferencia es de casi -11 puntos porcentuales (o de casi un $40 \%$ con respecto a la media de la categoría base) en el caso del empleador hombre y de casi -8pp (30\%) en el caso de la empleadora mujer.

En suma, las trabajadoras mujeres tienen menos probabilidad de postular si el empleador revela el género (tanto masculino como femenino) con respecto al caso del empleador de género neutro. Esta brecha se encuentra entre el $20 \%$ y el $40 \%$, en promedio, siendo mayor en el contexto en el cual el precio es no negociable.

Este resultado es contraintuitivo si se considera que el empleador de género neutro representa la ausencia de información sobre el género, dado que la literatura sostiene que, en general, la ambiguedad juega en contra de los resultados laborales de las mujeres (Leibbrandt y List, 2015). Sin embargo, resulta relevante preguntarse si al recibir una oferta de empleo de un representante de una companía que no revela el género los freelancers infieren que se trata de un empleador de un determinado género (hombre o mujer) recurriendo a priors sobre probabilidades ex antes.

Por último, las diferencias en tasa de respuesta entre empleador hombre y empleadora mujer no son significativas en ninguno de los contextos de negociación. 
Figura 3: Brecha \% en respuesta con empleador neutro

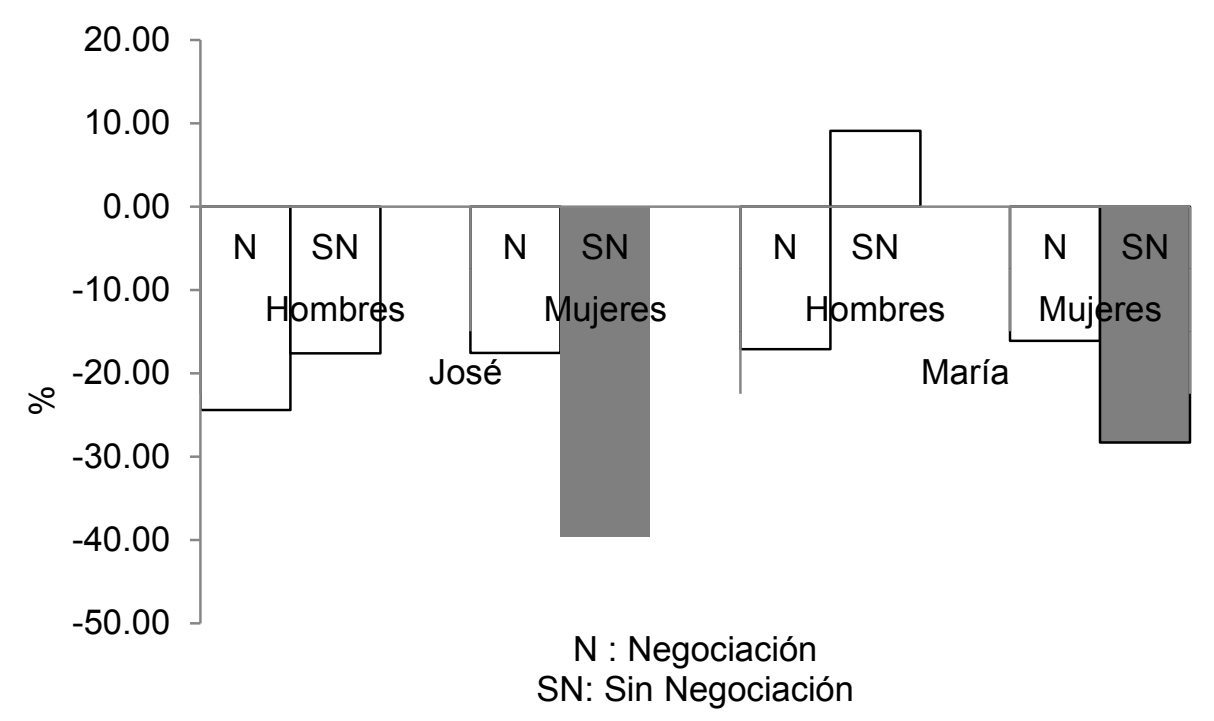

\subsection{Salarios}

La remuneración pretendida representada por monto o salario al cual ofrecen realizar el trabajo los freelancers es una señal más informativa sobre su voluntad de negociar o competir por precio, con respecto a la tasa de respuesta analizada en la sección anterior.

Es importante notar que, incluso con asignación aleatoria, las brechas causales en monto son más difíciles de identificar debido a la posible selección muestral. En particular, solo observamos remuneración pretendida para aquellos freelancers que han decidido postular al trabajo. La intuición detrás de este razonamiento se basa en el hecho de que aquellos freelancers que han decidido postular son potencialmente diferentes de aquellos que no, al interior de cada celda de tratamiento (e.g. candidatos que postulan versus candidatos que no postulan al empleador de género $g$ ) y entre tratamientos (e.g. candidatos que postulan al empleador de género $h$ versus aquellos que postulan al empleador de género $m$ ). Este problema podría verse agravado por la baja tasa de respuesta que obtenemos en el experimento en general (18\%).

En economía experimental, la validez de la interpretación de los resultados va a depender del supuesto de que la no-respuesta es aleatoria, es decir, no está correlacionada con la asignación del tratamiento o con el resultado de interés (Heckman, 1974).

En nuestro caso, es posible que las ofertas observadas (monto o salario) estén correlacionadas con la probabilidad de aceptar una invitación de empleo de un determinado empleador. El hecho de que no encontramos diferencias significativas en tasa de respuesta entre nuestro empleador hombre (José) y nuestra empleadora mujer (María) es evidencia a favor del supuesto mencionado anteriormente, aunque no conclusiva. En cualquier caso, las estimaciones pueden corregirse econométricamente utilizando el método de cotas (o bounds) de Lee (2008), por ejemplo. En esta etapa del trabajo nos limitamos a presentar resultados experimentales sin las mencionadas correcciones.

Estos resultados (tabla 2) muestran que tanto freelancers hombres como freelancers mujeres piden menores salarios, por el mismo trabajo, si el empleador es hombre. En promedio, José, nuestro empleador hombre, recibe ofertas por 33 euros menos en el contexto de negociación, cantidad que 
representa una diferencia alrededor de un $22 \%$ con respecto a la empleadora mujer y al empleador de género neutro.

Por otra parte, es importante notar que la empleadora mujer y el empleador neutro resultan equivalentes en términos de remuneración pretendida: las diferencias en monto entre ambos tipos de empleadores no solo no son significativas sino que, además, son pequeñas en magnitud (entre 1 y 5 euros).

En conjunto, todo esto estaría indicando que el efecto encontrado representa un "efecto empleador hombre", el cual es virtualmente idéntico para trabajadores de ambos sexos.

Las figuras 4a y 4b muestran la proporción de los 250 euros (precio máximo ofrecido) que los candidatos potenciales piden a cada uno de los empleadores. Por ejemplo, observamos que los freelancers hombres piden $50 \%$ del salario al empleador hombre, mientras que piden $65 \%$ del salario disponible a la empleadora mujer.

Figura 4: Excedente del empleador en contexto negociable

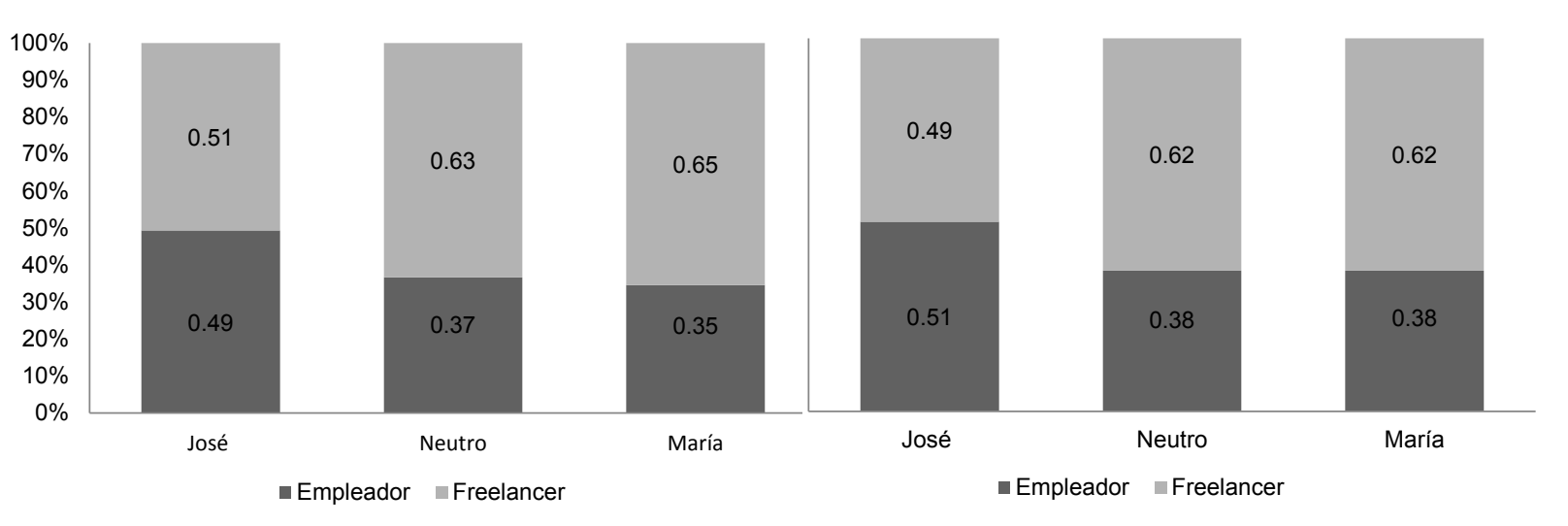

(a) Freelancers hombres

(b) Freelancers mujeres

En cuanto a las diferencias en monto entre contextos de negociación, es de esperar que las ofertas recibidas en el contexto en que ofrecemos un salario de 250 euros, no negociables, sean mayores que aquellas que recibimos en el contexto en el cual este precio es negociable en el rango 25-250 euros, a pesar de que se trata de exactamente el mismo trabajo.

En este último caso, además de ofrecer negociación, estamos indicando una cota inferior de 25 euros que puede sesgar las ofertas hacia esa dirección. Aún conciendo la utilidad de reserva del empleador (e.g. cota superior), los freelancers van a preferir pedir una menor compensación salarial a cambio de incrementar sus probabilidades de obtener el empleo.

En efecto, el $90 \%$ de las propuestas recibidas en el contexto donde el salario no es negociable representan exactamente la cantidad ofrecida por el empleador: 250 euros (siendo la diferencia promedio en monto entre ambos tipos de contextos de 96 euros, aproximadamente).

Sin embargo, esta proporción difiere por género del empleador. Más específicamente, si la oferta proviene de una empleadora mujer, la diferencia en excedente potencial para el empleador entre ambos contextos de negociación cae de 96 a 86 euros (debido a los mayores salarios que ya le pedían en el contexto negociable). Por otra parte, si la oferta de empleo proviene de un empleador hombre, la diferencia aumenta de 96 a 110 euros. 
Solo el $84 \%$ de las propuestas al empleador hombre en el contexto de precio no negociable son de 250 euros. Esto implica que los freelancers tienen mayor probabilidad de competir por precio, incluso en el contexto en el cual este es fijo, con el empleador de género masculino. Este último efecto es significativo e importante en magnitud únicamente en el caso de los trabajadores hombres (24 euros menos al empleador hombre, con respecto al empleador de género neutro y a la empleadora mujer; una diferencia porcentual de un $9 \%$ ).

Figura 5: Excedente del empleador en contexto no negociable

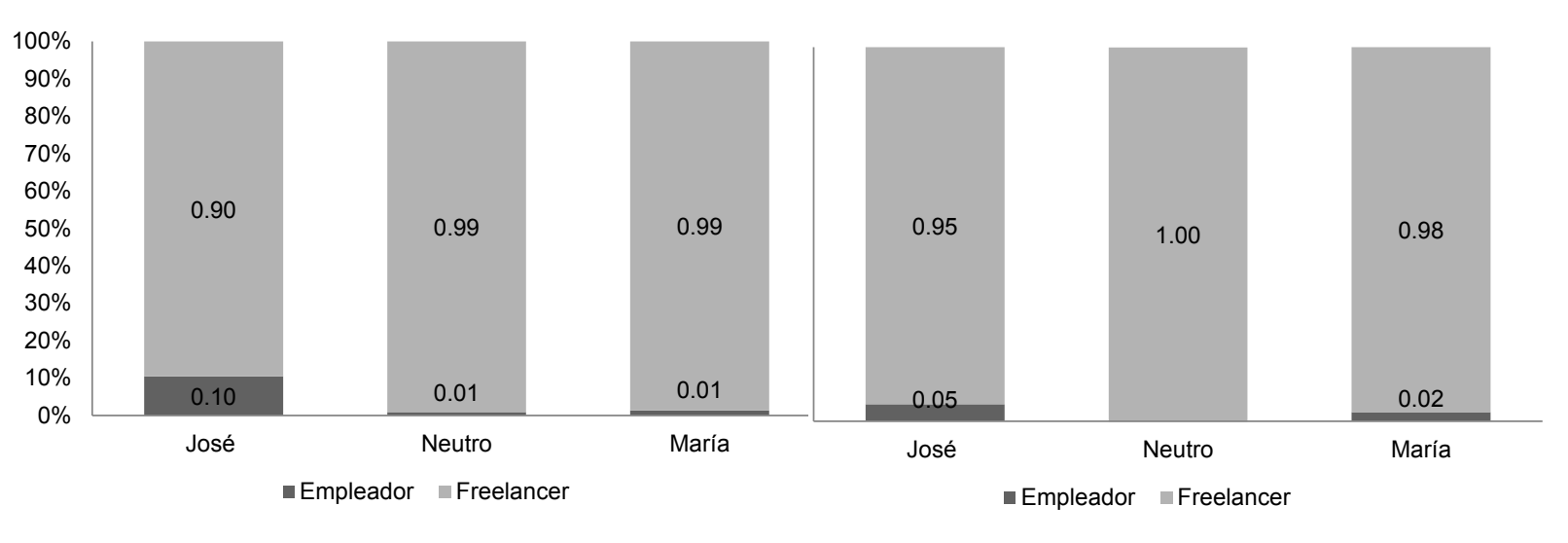

(a) Freelancers hombres

(b) Freelancers mujeres

\section{Interpretación y mecanismos}

\subsection{Marco teórico}

Una vez identificado este efecto empleador hombre sobre las remuneraciones pretendidas de los candidatos, una segunda etapa del proyecto consiste en intentar explicarlo, postulando hipótesis y testeando potenciales mecanismos subyacentes.

Una posible explicación podría tener que ver con el hecho de que los freelancers ofrecen productos diferenciados a empleadores de distinto género y, por lo tanto, piden precios diferentes.

¿Por qué ofrecerían, sistemáticamente, productos diferentes a hombres y a mujeres? La decisión del freelancer puede descomponerse en dos etapas. En primer lugar, el freelancer decide si acepta o no la invitación a postular al trabajo. En segundo lugar, si decide postular, debe indicar su remuneración pretendida. Este proceso podría modelarse utilizando la teoría básica del consumidor.

La utilidad esperada del freelancer viene dada por:

$$
U E_{F}=U E_{F}\left[p, e(q, t),\left(\omega_{F p}, \omega_{F e}\right)\right]=\left(\omega_{F p} p-\omega_{F e} e(q, t)\right) P(\text { ganar })
$$

Donde $p$ indica el precio que recibirá por el trabajo y $e(q, t)$ el nivel de esfuerzo que deberá realizar, el cual es función decreciente del tiempo de entrega $t$ (e.g. a mayor plazo otorgado, menor esfuerzo) y creciente en la calidad $q$. Lo importante de esta función de utilidad es va a contener un vector de ponderadores de precio y esfuerzo, denotado por $\left(\omega_{F p}, \omega_{F e}\right)$. Este vector indica cuánto 
valora el freelancer el precio o salario y qué tanto valora (o descuenta) el esfuerzo que debe realizar.

Se podría suponer, además, que realizar el trabajo rápido es menos costoso que hacerlo bien:

$$
\omega_{F e}=\left(\omega_{F t}, \omega_{F q}\right): \omega_{t}<\omega_{q}
$$

El freelancer va a maximizar esta función de utilidad esperada de dos maneras. Por un lado, intentará incrementar la brecha ponderada entre el tiempo y el esfuerzo o $\omega_{F p} p-\omega_{F e} e(q, t)$ tanto como pueda: pidiendo un mayor $p$ (o remuneración pretendida) y ofreciendo un menor $e(q, t)$ (mayor plazo de entrega o menor calidad). Por otro lado, también deberá maximizar su probabilidad de ganar el trabajo.

Esta probabilidad de ganar depende positivamente de la función de utilidad esperada del empleador, tal cual la percibe el freelancer y de un factor estocástico $\epsilon(N)$ que puede depender del número de competidores, entre otras cosas:

$$
P(\text { ganar })=P\left[U E_{E}\left(p, e(q, t),\left(\omega_{E p}, \omega_{E e}\right)\right), \epsilon(N)\right]
$$

Entonces, para maximizar este segundo componente, la probabilidad de obtener el empleo o "ganar", la estrategia del freelancer consiste en maximizar la utilidad percibida del empleador, sujeto a que está maximizando también la suya.

Cuando el freelancer realiza su oferta, ya se encuentra en su utilidad de reserva: la utilidad mínima que estaría dispuesto a recibir en caso de obtener el trabajo. Es decir, a estas alturas, podemos abstraernos de los ponderadores de la utilidad del freelancer, puesto que ya fue revelada. Puesto de otra forma, el freelancer postula y ofrece un precio siempre que:

$$
\left(\omega_{F p} p-\omega_{F e} e(q, t)\right) P(\text { ganar }) \geq \underline{U}=U(\text { No postular })
$$

Nos concentramos, entonces, en la segunda etapa del proceso de maximización de la utilidad esperada del freelancer: su estrategia para maximizar su probabilidad de ganar o $P($ ganar $)$.

Para maximizar esta probabilidad, tiene que maximizar la utilidad esperada del empleador y, para ello, debe primero darle forma:

$$
\begin{gathered}
U E_{E}\left[p, e(q, t),\left(\omega_{E p}, \omega_{E e}\right)\right]=\omega_{E e} e(q, t)-\omega_{E p} p \\
\omega_{E e}=\left(\omega_{E t}, \omega_{E q}\right)
\end{gathered}
$$

La estrategia final del freelancer, la cual se traduce en la oferta que observamos, va a estar formada por un precio $p$, una oferta de calidad $q$ y un tiempo de entrega $t$ que, dado que ya maximizan su utilidad (dejándolo, por ejemplo, en su utilidad de reserva), van a estar maximizando también la función de utilidad del empleador y, por ende, la probabilidad de ganar el trabajo:

$$
S_{F}=(p, t, q)
$$

Para obtener $S_{F}$, el freelancer tiene que inferir, a través de la información que tiene disponible, los valores relativos de los ponderadores de la función de utilidad del empleador, es decir, cuánto valora el precio que debe pagar (salario) y el esfuerzo como función del tiempo de entrega y la calidad. 


\subsection{Hipótesis y tests}

Es importante observar que los ponderadores $\left(\omega_{E p}, \omega_{E t}, \omega_{E q}\right)$ no necesariamente son los ponderadores reales en la función de utilidad de los empleadores, sino los percibidos por los freelancers. Es esta percepción o formación de creencias la que determina, en última instancia, el comportamiento o estrategia.

Si estos ponderadores percibidos dependiesen del género del empleador, luego de maximizar su utilidad esperada, los freelancers podrían terminar ofreciendo un trabajo diferenciado a empleadores hombres y mujeres:

$$
\left(\omega_{E p}, \omega_{E t}, \omega_{E q}\right)=\left(\omega_{E p}(g), \omega_{E t}(g), \omega_{E q}(g)\right)
$$

En particular, si para la empleadora mujer:

$$
\begin{aligned}
& \omega_{E p}(m)<\omega_{E q}(m) \\
& \omega_{E t}(m)<\omega_{E q}(m)
\end{aligned}
$$

Y para el empleador hombre:

$$
\begin{aligned}
& \omega_{E p}(h)>\omega_{E q}(h) \\
& \omega_{E t}(h)>\omega_{E q}(h)
\end{aligned}
$$

Los freelancers terminarían ofrenciendo un producto de mayor calidad a la empleadora mujer y, en cambio, un trabajo más rápido y simple al empleador hombre. Este comportamiento se reflejaría, además, en un mayor precio en el primer caso: diferencial que compensaría el mayor esfuerzo que esperaría realizar el freelancer.

Es decir, la estrategia $S_{F}=(p, t, q)$ sería diferente ante empleadores hombres y mujeres:

$$
S_{F}=(p, t, q)=\left(p\left(\omega_{E p}, \omega_{F p}\right),\left(\omega_{E t}, \omega_{F t}\right), t\left(\omega_{E q}, \omega_{F q}\right)\right.
$$

En la práctica, esta hipótesis podría testearse de tres maneras:

(1) Efecto composición: analizando el pool de freelancers que postulan a cada empleador. Deberíamos observar que los freelancers que postulan a la oferta del empleador hombre tienen un costo de ofrecer calidad más alto (por ejemplo, tienen menos experiencia o credenciales, en promedio).

Esto tiene que ver con el hecho de que el freelancer maximizó su utilidad esperada antes de postular. Van a querer maximizar la utilidad esperada de cada empleador siempre que les convenga aceptar la invitación. El empleador hombre, cuyo $\omega_{E q}$ es relativamente bajo, va a recibir ofertas de freelancers que ponderan más negativamente el componente del esfuerzo "calidad". La empleadora 
mujer, en cambio, cuyo $\omega_{E q}$ es relativamente alto, recibirá una mayor proporción de ofertas de freelancers que ponderan menos negativamente la calidad.

(2) Estrategias: analizando, de manera directa, la forma en que postulan los freelancers a cada empleador. Es decir, estudiando las ofertas textuales de los freelancers y qué ofrecen -o no ofrecenen cada caso. Deberíamos observar que los freelancers ofrecen calidad a la empleadora mujer y solo precio o entrega rápida al empleador hombre (en promedio y en términos relativos).

Autores como Ng y Leung (2015) recurren a técnicas de análisis linguístico cuantitativo del texto de propuestas para explorar diferencias en las formas en que hombres y mujeres se comportan cuando postulan a un empleo. En este sentido, sostienen que los candidatos pueden enfatizar tanto los aspectos relacionales como transaccionales del trabajo, lo cual tiene un impacto sobre la probabilidad de contratación. Mientras que la estrategia relacional, la más utilizada por las mujeres en el estudio, consiste en establecer una conexión social con el empleador, la estrategia transaccional, la más utilizada por los hombres, se enfoca en aspectos cuantitativos y pecunarios. Se podría incorporar esta metodología para identificar diferencias en la forma en que los freelancers postulan a ofertas de empleo de trabajadores hombres y mujeres y testear así la hipótesis presentada en esta sección.

(3) Tratamientos adicionales (de información): variando los parámetros $\omega_{E p}, \omega_{E t}, \omega_{E q}$ que perciben los freelancers (o "tipos" de empleadores). En este caso, al proveer información relevante, los estereotipos perderían importancia y el género del empleador debería de ser un factor clave en la estrategia.

\section{Comentarios finales}

La participación y la negociación son dos determinantes fundamentales de los resultados en el mercado laboral. Las diferencias por género en estas etapas iniciales podrían explicar gran parte de la brecha salarial observada. Debido a cuestiones de auto-selección, resulta difícil separar estos determinantes de otras características de los trabajadores en contextos laborales reales.

Es por ello que diseñamos un experimento de campo en una plataforma de empleo en línea real, cuyos participantes ignoran que observamos su comportamiento. Nuestro diseño experimental nos

permite estudiar diferencias de género en participación (e.g. voluntad de postular a un trabajo en un contexto de salario negociable o no negociable) y negociación (e.g. voluntad de negociar un mayor salario o competir por precio).

Más específicamente, nos preguntamos si estas diferencias dependen del género del empleador. Para ello, aleatorizamos una muestra de freelancers hombres y mujeres a distintas ofertas laborales, todas idénticas excepto por dos factores: el genero del empleador y la posibilidad de negociar o no el salario. Encontramos que tanto hombres como mujeres ofrecen realizar los mismos trabajos por un salario hasta un $22 \%$ menor si el empleador es hombre.

Por otra parte, no encontramos diferencias en preferencias por contextos de negociación, aunque los freelancers tienen una mayor probabilidad de competir por precio cuando la alternativa de negociación es explícita y pedir el precio ofrecido cuando esta opción se proscribe. Los freelancers hombres, por su parte, son más propensos a competir por precio incluso en un contexto donde el salario no es negociable. Este resultado es consistente con la literatura que encuentra una desventaja en competencia para las mujeres (e.g., Gneezy et al., 2003).

Una pregunta crucial que surge de nuestro estudio es por qué los freelancers tratan diferencialmente a los empleadores según su género. En particular, por qué un empleador de género masculino 
tendría ventajas en negociación respecto a una empleadora femenina o de género neutro, en términos de excedente potencial.

Las explicaciones podrían basarse, por un lado, en la existencia de discriminación pura: es decir, gustos o preferencias de los freelancers. En este caso, los freelancers podrían estar cobrándole un premio a la empleadora mujer. Esta alternativa es poco plausible, dada la equivalencia que encontramos entre la empleadora mujer y el empleador de género neutro.

Por otro lado, podríamos encontrarnos ante un problema de discriminación estadística: es decir, el efecto podría explicarse a través de las expectativas de los freelancers acerca de los empleadores en términos de probabilidades de contratación, cumplimiento del contrato, competencia o alternativas externas, ventajas en negociación, gustos y preferencias, entre otros factores. En particular, los freelancers podrían esperar que los empleadores hombres sean más duros negociando o ponderen más la competencia por precio que otros atributos de los candidatos. La sección 5 brinda un marco teórico que hace plausible testear esta última hipótesis.

Nuestros resultados brindan información acerca de los determinantes salariales en tanto identifican y cuantifican la importancia de las interacciones de género en cuestiones de participación y negociación en el mercado laboral. Asimismo, testeamos nuestras hipótesis en un contexto en el cual la información tiene un valor marginal alto como determinante del matching y la distribución del excedente: las plataformas de empleo en línea. Estas plataformas son, además, representativas de nuevas formas impersonales de interacción económica en las cuales las características de los agentes deben inferirse de muy poca información disponible. Nuestras estimaciones podrían, en este sentido, representar un lower bound, si esperamos que el efecto se incremente en interacciones cara a cara. 


\section{Referencias}

Arceo-Gomez, E., y Campos-Vazquez, R. (2013). Race and marriage in the labor market: A discrimination correspondence study in a developing country. Working Paper MPRA.

Azmat, B., Ghazala y Petrongolo. (2014). Gender and the labor market: What have we learned from field and lab experiments? Labour Economics, 30, 32-40.

Bertrand, M. (2011). New perspectives on gender. Handbook of Labor Economics, 4 (17), 1545-1592.

Bertrand, M., y Duflo, E. (2016). Field experiments on discrimination. Working Paper $N B E R(22014)$.

Bertrand, M., Kamenica, E., y Pan, J. (2015). Gender identity and relative income within households. The Quarterly Journal of Economics, 571-614.

Booth, A., y Leigh, A. (2010). Do employers discriminate by gender? a field experiment in femaledominated occupations. Discussion Paper IZA(4690).

Card, D., Cardoso, A., y Kline, P. (2015). Bargaining, sorting and the gender wage gap: Quantifying the impact of firms on the relative pay of women. The Quarterly Journal of Economics.

Chen, C., Kast, F., y Pomeranz, D. (2009). Shedding light on the gender gap through randomized field studies. Working Paper WAPPP.

Chen, Z., Ong, D., y Sheremeta, R. (2015). The gender difference in the value of winning. Working Paper.

Cullen, Z., y Pakzad-Hurson, B. (2014). Equal pay for equal work: Evidence from the renegotiation of short-term contracts. Job Market Paper.

Gasparini, L., y Marchionni, M. (2015). Bridging gender gaps? the rise and deceleration of female labor force participation in latin america.

Glennerster, R., y Takavarasha, K. (2013). Running randomized evaluations: A practical guide.

Goldin, C. (2002). A pollution theory of discrimination: Male and female differences in occupations and earnings. Working Paper NBER(8985).

Goldin, C. (2006). The quiet revolution that transformed women's employment, education and family. Working Paper NBER(11953).

Ibanez, M., Rai, A., y Gerhard, R. (2015). Sorting throguh affirmative action: Three field experiments in colombia. Discussion Paper Dusseldorf Institute for Competition Economics(183).

Janssen, S., Tuor Sartore, S., y Backes-Gellner, U. (2014). Social attitudes on gender equality and firms' discriminatory pay-setting. Working Paper IZA(7959).

Leibbrandt, A., y List, J. (2015). Do women avoid salary negotiations? evidence from a large scale natural field experiment. Management Science, 61(9), 2016-2024.

List, J., y Rasul, I. (2011). Field experiments in labor economics. Handbook of Labor Economics(2), 103-228.

Neumark, D. (2016). Experimental research on labor market discrimination. Working Paper $\operatorname{NBER}(22022)$.

Ng, W., y Leung, M. (2015). For love or money? gender differences in how one approaches getting a job. Working Paper.

Pallais, A. (2013). Inefficient hiring in entry-level labor markets. Working Paper NBER.

Stanton, C., y Thomas, C. (2014). Landing the first job: The value of intermediaries in online hiring. Working Paper. 
Tabla 1: Resultados desagregados por género del empleador y contexto de negociación: Tasa de respuesta

Tabla I A: Tasa de respuesta por género del empleador y contexto de negociación

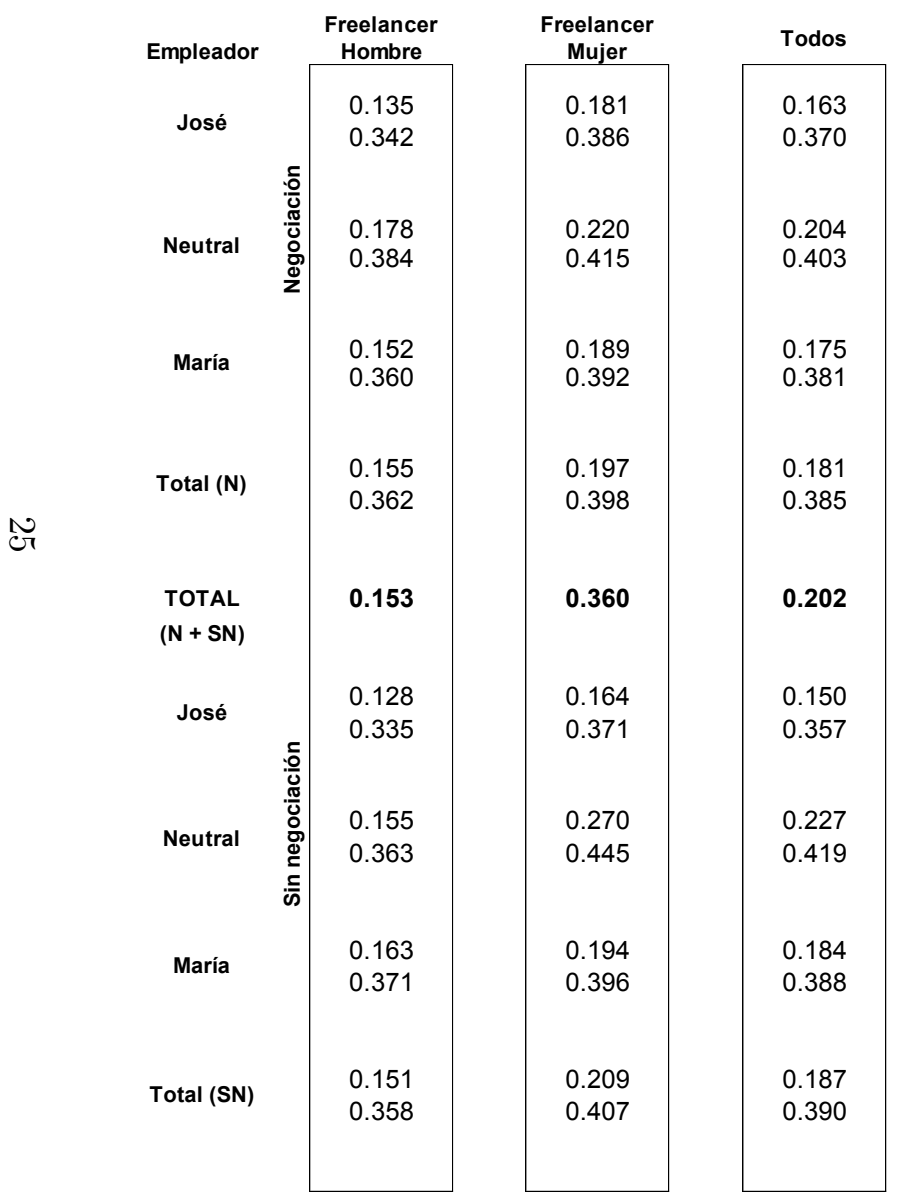

Tabla I B: Diferencias en tasa de respuesta por género del empleador y contexto de negociacion

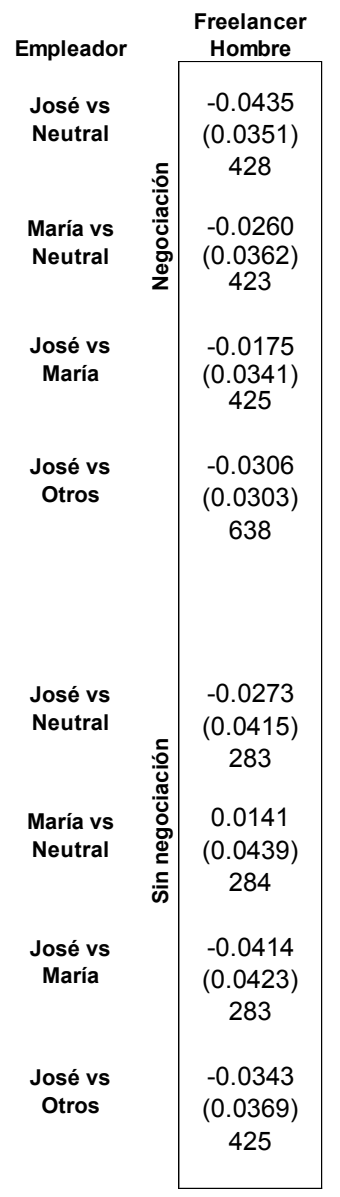

Tabla I C: Diferencias en tasa de respuesta por contexto de negociación y género del empleador

\begin{tabular}{c|c|}
\multicolumn{1}{c}{ Empleador } & \multicolumn{1}{c}{$\begin{array}{c}\text { Freelancer } \\
\text { Hombre }\end{array}$} \\
\cline { 2 - 3 } José & 0.00722 \\
N vs SN & $(0.0368)$ \\
& 356 \\
& \\
Neutral & 0.0235 \\
N vs SN & $(0.0407)$ \\
& 355 \\
María & -0.0166 \\
N vs SN & $\begin{array}{c}0.0398) \\
\end{array}$ \\
Total & 0.00458 \\
N vs SN & $(0.0226)$ \\
& 1,063 \\
&
\end{tabular}

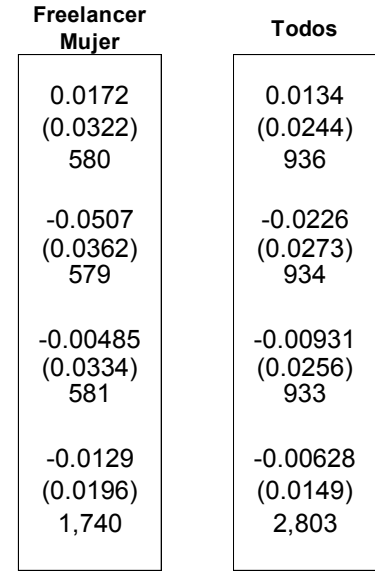

Tabla I D: tasa de respuesta total (N + SN)

\begin{tabular}{|c|c|c|}
\hline $\begin{array}{l}\text { José vs } \\
\text { Neutral }\end{array}$ & $\begin{array}{c}-0.0370 \\
(0.0268) \\
711\end{array}$ & $\begin{array}{c}-0.0659^{\star \star \star} \\
(0.0237) \\
1,159\end{array}$ \\
\hline $\begin{array}{l}\text { María vs } \\
\text { Neutral }\end{array}$ & $\begin{array}{c}-0.00992 \\
(0.0279) \\
707\end{array}$ & $\begin{array}{c}-0.0490^{\star *} \\
(0.0241) \\
1,160\end{array}$ \\
\hline $\begin{array}{c}\text { José vs } \\
\text { María }\end{array}$ & $\begin{array}{c}-0.0271 \\
(0.0265) \\
708\end{array}$ & $\begin{array}{c}-0.0169 \\
(0.0227) \\
1,161\end{array}$ \\
\hline $\begin{array}{l}\text { José vs } \\
\text { Otros }\end{array}$ & $\begin{array}{c}-0.0321 \\
(0.0234) \\
1,063\end{array}$ & $\begin{array}{c}-0.0414^{* *} \\
(0.0204) \\
1,740\end{array}$ \\
\hline
\end{tabular}

$$
\begin{gathered}
-0.0549^{\star \star *} \\
(0.0179) \\
1,870 \\
\\
-0.0341^{\star} \\
(0.0184) \\
1,867 \\
\\
-0.0209 \\
(0.0173) \\
1,869 \\
\\
-0.0379^{\star *} \\
(0.0155) \\
2,803
\end{gathered}
$$


Tabla 2: Resultados desagregados por género del empleador y contexto de negociación: Monto (salario)

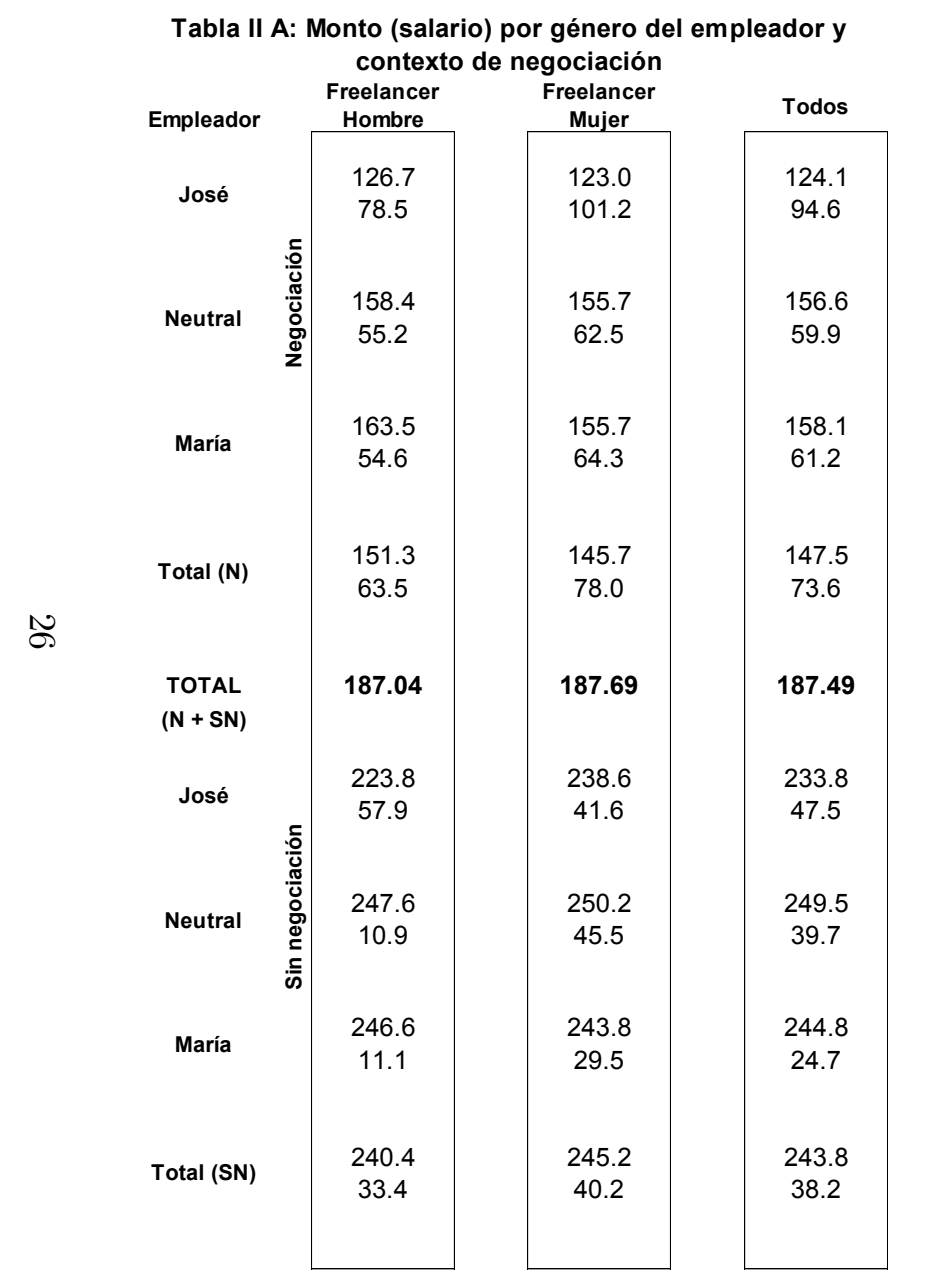

Tabla II B: Diferencias en monto (salario) por género del empleador y contexto de negociación

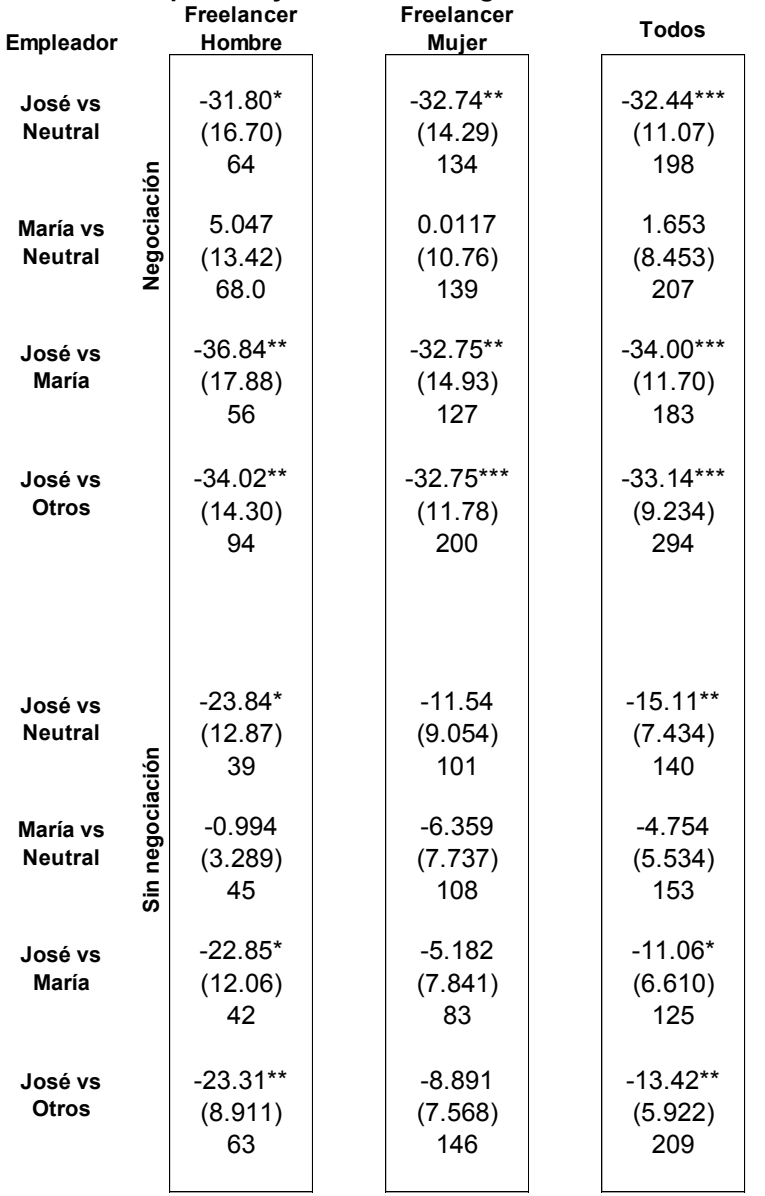

Tabla II C: Diferencias en monto (salario) por contexto de negociación y género del empleador

\begin{tabular}{|c|c|c|c|}
\hline Empleador & $\begin{array}{l}\text { Freelancer } \\
\text { Hombre }\end{array}$ & $\begin{array}{l}\text { Freelancer } \\
\text { Mujer }\end{array}$ & Todos \\
\hline $\begin{array}{c}\text { José } \\
\text { N vs SN }\end{array}$ & $\begin{array}{c}-97.13^{\star * *} \\
(21.74) \\
44\end{array}$ & $\begin{array}{c}-115.7^{\star \star *} \\
(17.29) \\
99\end{array}$ & $\begin{array}{c}-109.9^{\star * *} \\
(13.68) \\
143\end{array}$ \\
\hline $\begin{array}{l}\text { Neutral } \\
\mathrm{N} \text { vs SN }\end{array}$ & $\begin{array}{c}-89.17^{\star * *} \\
(12.22) \\
59\end{array}$ & $\begin{array}{c}-94.46^{* * *} \\
(9.507) \\
136\end{array}$ & $\begin{array}{c}-92.95^{\star * *} \\
(7.600) \\
195\end{array}$ \\
\hline $\begin{array}{c}\text { María } \\
\mathrm{N} \text { vs SN }\end{array}$ & $\begin{array}{c}-83.13^{* * *} \\
(11.35) \\
54\end{array}$ & $\begin{array}{c}-88.09^{* \star *} \\
(10.26) \\
111\end{array}$ & $\begin{array}{c}-86.44^{* * *} \\
(7.814) \\
165\end{array}$ \\
\hline $\begin{array}{c}\text { Total } \\
\text { N vs SN }\end{array}$ & $\begin{array}{c}-89.16^{\star \star *} \\
(8.722) \\
157\end{array}$ & $\begin{array}{c}-99.48^{\star \star *} \\
(7.054) \\
346\end{array}$ & $\begin{array}{c}-96.29^{\star \star \star *} \\
(5.567) \\
503\end{array}$ \\
\hline \multicolumn{4}{|c|}{ Tabla II D: Monto (salario) total (N+SN) } \\
\hline $\begin{array}{l}\text { José vs } \\
\text { Neutral }\end{array}$ & $\begin{array}{c}-23.80 \\
(14.49) \\
103\end{array}$ & $\begin{array}{c}-32.10^{* * *} \\
(11.30) \\
235\end{array}$ & $\begin{array}{c}-29.60^{* * *} \\
(8.992) \\
338\end{array}$ \\
\hline $\begin{array}{l}\text { María vs } \\
\text { Neutral }\end{array}$ & $\begin{array}{c}10.25 \\
(11.36) \\
113\end{array}$ & $\begin{array}{c}-8.033 \\
(9.048) \\
247\end{array}$ & $\begin{array}{c}-2.277 \\
(7.152) \\
360\end{array}$ \\
\hline $\begin{array}{l}\text { José vs } \\
\text { María }\end{array}$ & $\begin{array}{c}-34.06^{\star *} \\
(14.55) \\
98\end{array}$ & $\begin{array}{c}-24.07^{\star *} \\
(11.77) \\
210\end{array}$ & $\begin{array}{c}-27.32^{* * *} \\
(9.243) \\
308\end{array}$ \\
\hline $\begin{array}{l}\text { José vs } \\
\text { Otros }\end{array}$ & $\begin{array}{c}-28.70^{\star *} \\
(12.10) \\
157\end{array}$ & $\begin{array}{c}-28.49^{* \star *} \\
(9.561) \\
346\end{array}$ & $\begin{array}{c}-28.55^{\star * *} \\
(7.573) \\
503\end{array}$ \\
\hline
\end{tabular}


Tabla 3: Regresiones con controles pre-tratamiento

\begin{tabular}{|c|c|c|c|c|c|c|c|c|}
\hline \multirow{4}{*}{$\begin{array}{l}\text { Variables } \\
\text { José }\end{array}$} & \multirow{2}{*}{\multicolumn{4}{|c|}{$\begin{array}{l}\text { Tasa de respuesta } \\
\text { Freelancers hombres }\end{array}$}} & & & & \\
\hline & & Freelan & & & \multicolumn{4}{|c|}{ Freelancers mujeres } \\
\hline & \multicolumn{2}{|c|}{ Negociación } & \multicolumn{2}{|c|}{ Sin negociación } & \multicolumn{2}{|c|}{ Negociación } & \multicolumn{2}{|c|}{ Sin negociación } \\
\hline & $\begin{array}{c}-0.0435 \\
(0.0350)\end{array}$ & $\begin{array}{l}-0.0318 \\
(0.0346)\end{array}$ & $\begin{array}{l}-0.0273 \\
(0.0426)\end{array}$ & $\begin{array}{c}-0.0466 \\
(0.0424)\end{array}$ & $\begin{array}{c}-0.0386 \\
(0.0302)\end{array}$ & $\begin{array}{l}-0.0424 \\
(0.0300)\end{array}$ & $\begin{array}{c}-0.107^{* * *} \\
(0.0376)\end{array}$ & $\begin{array}{c}-0.106^{\star \star *} \\
(0.0376)\end{array}$ \\
\hline María & $\begin{array}{c}-0.0260 \\
(0.0352)\end{array}$ & $\begin{array}{l}-0.0176 \\
(0.0347)\end{array}$ & $\begin{array}{c}0.0141 \\
(0.0425)\end{array}$ & $\begin{array}{l}0.00161 \\
(0.0421)\end{array}$ & $\begin{array}{c}-0.0305 \\
(0.0302)\end{array}$ & $\begin{array}{l}-0.0377 \\
(0.0300)\end{array}$ & $\begin{array}{c}-0.0764^{* *} \\
(0.0376)\end{array}$ & $\begin{array}{c}-0.0751^{* *} \\
(0.0376)\end{array}$ \\
\hline \multicolumn{9}{|l|}{ Controles } \\
\hline Tiempo de envío de propuesta & $x$ & $\begin{array}{c}-0.0170 \\
(0.0285)\end{array}$ & $x$ & $\begin{array}{c}-0.0354 \\
(0.0451)\end{array}$ & $x$ & $\begin{array}{c}-0.00886 \\
(0.0202)\end{array}$ & $x$ & $\begin{array}{l}0.0471^{* *} \\
(0.0238)\end{array}$ \\
\hline Valor promedio de propuestas & $\mathrm{x}$ & $\begin{array}{l}0.00328 \\
(0.0118)\end{array}$ & $\mathrm{x}$ & $\begin{array}{c}-0.0243 \\
(0.0150)\end{array}$ & $\mathrm{x}$ & $\begin{array}{l}-0.00977 \\
(0.00949)\end{array}$ & $\mathrm{x}$ & $\begin{array}{c}-0.0143 \\
(0.0126)\end{array}$ \\
\hline Valor promedio de proyectos & $x$ & $\begin{array}{l}-0.00438 \\
(0.0165)\end{array}$ & $x$ & $\begin{array}{c}0.000309 \\
(0.0204)\end{array}$ & $x$ & $\begin{array}{l}-0.00588 \\
(0.0142)\end{array}$ & $x$ & $\begin{array}{l}0.00571 \\
(0.0185)\end{array}$ \\
\hline Total de propuestas enviadas & $x$ & $\begin{array}{c}0.0538^{* * *} \\
(0.0135)\end{array}$ & $x$ & $\begin{array}{c}0.0531^{\star * *} \\
(0.0164)\end{array}$ & $x$ & $\begin{array}{c}0.0480^{\star * *} \\
(0.0122)\end{array}$ & $x$ & $\begin{array}{r}-0.00149 \\
(0.0151)\end{array}$ \\
\hline Fecha de registro del freelancer & $x$ & $\begin{array}{l}0.0420^{* *} \\
(0.0176)\end{array}$ & $x$ & $\begin{array}{c}0.0120 \\
(0.0199)\end{array}$ & $x$ & $\begin{array}{c}0.0178 \\
(0.0144)\end{array}$ & $x$ & $\begin{array}{c}0.0248 \\
(0.0175)\end{array}$ \\
\hline Constante & $\begin{array}{l}0.178^{* * *} \\
(0.0248)\end{array}$ & $\begin{array}{c}0.0231 \\
(0.0890)\end{array}$ & $\begin{array}{l}0.155^{\star * *} \\
(0.0301)\end{array}$ & $\begin{array}{c}0.173 \\
(0.109)\end{array}$ & $\begin{array}{l}0.220^{* * *} \\
(0.0214)\end{array}$ & $\begin{array}{c}0.184^{*} \\
(0.0972)\end{array}$ & $\begin{array}{l}0.270^{* * *} \\
(0.0266)\end{array}$ & $\begin{array}{c}0.137 \\
(0.107)\end{array}$ \\
\hline Media de VD & $\begin{array}{l}0.1552 \\
0.3623\end{array}$ & $\begin{array}{l}0.1552 \\
0.3623\end{array}$ & $\begin{array}{l}0.1506 \\
0.3581\end{array}$ & $\begin{array}{l}0.1506 \\
0.3581\end{array}$ & $\begin{array}{l}0.1965 \\
0.3976\end{array}$ & $\begin{array}{l}0.1965 \\
0.3976\end{array}$ & $\begin{array}{l}0.2095 \\
0.4072\end{array}$ & $\begin{array}{l}0.2095 \\
0.4072\end{array}$ \\
\hline $\begin{array}{l}N \\
\text { R2 }\end{array}$ & $\begin{array}{c}638 \\
0.002\end{array}$ & $\begin{array}{c}638 \\
0.042\end{array}$ & $\begin{array}{c}425 \\
0.002\end{array}$ & $\begin{array}{c}425 \\
0.040\end{array}$ & $\begin{array}{l}1,043 \\
0.002\end{array}$ & $\begin{array}{l}1,043 \\
0.022\end{array}$ & $\begin{array}{c}697 \\
0.012\end{array}$ & $\begin{array}{c}697 \\
0.022\end{array}$ \\
\hline
\end{tabular}

Monto o salario

\begin{tabular}{|c|c|c|c|c|c|c|c|c|}
\hline \multirow{3}{*}{$\begin{array}{l}\text { Variables } \\
\text { José }\end{array}$} & \multicolumn{4}{|c|}{ Freelancers hombres } & \multicolumn{4}{|c|}{ Freelancers mujeres } \\
\hline & \multicolumn{2}{|c|}{ Negociación } & \multicolumn{2}{|c|}{ Sin negociación } & \multicolumn{2}{|c|}{ Negociación } & \multicolumn{2}{|c|}{ Sin negociación } \\
\hline & $\begin{array}{c}-31.80^{* *} \\
(15.86)\end{array}$ & $\begin{array}{l}-31.67^{*} \\
(16.27)\end{array}$ & $\begin{array}{c}-23.84^{* *} \\
(10.35)\end{array}$ & $\begin{array}{c}-22.60^{\star *} \\
(10.81)\end{array}$ & $\begin{array}{c}-32.74^{\star *} \\
(13.34)\end{array}$ & $\begin{array}{c}-36.41^{* * *} \\
(13.29)\end{array}$ & $\begin{array}{l}-11.54 \\
(8.251)\end{array}$ & $\begin{array}{l}-10.34 \\
(8.378)\end{array}$ \\
\hline María & $\begin{array}{c}5.047 \\
(15.22) \\
\end{array}$ & $\begin{array}{c}2.817 \\
(15.43) \\
\end{array}$ & $\begin{array}{c}-0.994 \\
(9.626)\end{array}$ & $\begin{array}{c}1.942 \\
(10.28) \\
\end{array}$ & $\begin{array}{l}0.0117 \\
(13.06)\end{array}$ & $\begin{array}{c}0.337 \\
(13.08) \\
\end{array}$ & $\begin{array}{l}-6.359 \\
(7.841) \\
\end{array}$ & $\begin{array}{c}-5.463 \\
(7.949)\end{array}$ \\
\hline \multicolumn{9}{|l|}{ Controles } \\
\hline Tiempo de envío de propuesta & $x$ & $\begin{array}{c}10.43 \\
(13.78)\end{array}$ & $x$ & $\begin{array}{c}10.87 \\
(12.53)\end{array}$ & $x$ & $\begin{array}{c}13.98 \\
(9.003)\end{array}$ & $x$ & $\begin{array}{l}-3.786 \\
(4.920)\end{array}$ \\
\hline Valor promedio de propuestas & $x$ & $\begin{array}{c}10.48^{*} \\
(5.932)\end{array}$ & $x$ & $\begin{array}{l}-0.971 \\
(3.448)\end{array}$ & $x$ & $\begin{array}{l}11.17^{* *} \\
(4.573)\end{array}$ & $x$ & $\begin{array}{l}-3.582 \\
(2.772)\end{array}$ \\
\hline Valor promedio de proyectos & $x$ & $\begin{array}{c}2.908 \\
(8.355)\end{array}$ & $x$ & $\begin{array}{l}-5.110 \\
(5.848)\end{array}$ & $x$ & $\begin{array}{c}-4.192 \\
(6.707)\end{array}$ & $x$ & $\begin{array}{c}-0.831 \\
(3.970)\end{array}$ \\
\hline Total de propuestas enviadas & $x$ & $\begin{array}{c}6.479 \\
(5.929)\end{array}$ & $x$ & $\begin{array}{l}-0.210 \\
(3.699)\end{array}$ & $x$ & $\begin{array}{c}7.990 \\
(5.231)\end{array}$ & $x$ & $\begin{array}{c}0.333 \\
(3.242)\end{array}$ \\
\hline Fecha de registro del freelancer & $\mathrm{x}$ & $\begin{array}{c}-4.084 \\
(7.533) \\
\end{array}$ & $x$ & $\begin{array}{l}-1.107 \\
(4.521) \\
\end{array}$ & $\mathrm{x}$ & $\begin{array}{c}6.156 \\
(5.804) \\
\end{array}$ & $x$ & $\begin{array}{c}4.275 \\
(3.679)\end{array}$ \\
\hline Constante & $\begin{array}{l}158.4^{* * *} \\
(10.11)\end{array}$ & $\begin{array}{c}83.28^{*} \\
(44.01)\end{array}$ & $\begin{array}{c}247.6^{* * *} \\
(7.030)\end{array}$ & $\begin{array}{c}260.1^{* * *} \\
(28.88)\end{array}$ & $\begin{array}{l}155.7^{* * *} \\
(9.000)\end{array}$ & $\begin{array}{c}54.00 \\
(42.99)\end{array}$ & $\begin{array}{l}250.2^{* * *} \\
(5.061)\end{array}$ & $\begin{array}{c}268.7^{* * *} \\
(22.77)\end{array}$ \\
\hline Media de VD & $\begin{array}{c}151.3 \\
63.5\end{array}$ & $\begin{array}{c}151.3 \\
63.5\end{array}$ & $\begin{array}{c}240.4 \\
33.4\end{array}$ & $\begin{array}{c}240.4 \\
33.4\end{array}$ & $\begin{array}{c}145.7 \\
78.0\end{array}$ & $\begin{array}{c}145.7 \\
78.0\end{array}$ & $\begin{array}{c}245.2 \\
40.2\end{array}$ & $\begin{array}{c}245.2 \\
40.2\end{array}$ \\
\hline $\begin{array}{l}\mathrm{N} \\
\mathrm{R} 2\end{array}$ & $\begin{array}{c}94 \\
0.059 \\
\end{array}$ & $\begin{array}{c}94 \\
0.122 \\
\end{array}$ & $\begin{array}{c}63 \\
\mathbb{7} 701 \\
\end{array}$ & $\begin{array}{c}63 \\
0.137 \\
\end{array}$ & $\begin{array}{c}200 \\
0.038 \\
\end{array}$ & $\begin{array}{c}200 \\
0.095 \\
\end{array}$ & $\begin{array}{c}146 \\
0.014 \\
\end{array}$ & $\begin{array}{c}146 \\
0.044 \\
\end{array}$ \\
\hline
\end{tabular}


Tabla 4: Balance en variables pre-tratamiento

\begin{tabular}{|c|c|c|c|c|c|c|c|c|c|c|c|c|}
\hline & \multicolumn{6}{|c|}{ Freelancers hombres } & \multicolumn{6}{|c|}{ Freelancers mujeres } \\
\hline & María & $\mathrm{MxJ}$ & José & $\mathrm{JxN}$ & Neutro & $\mathrm{MxN}$ & María & $\mathrm{MxJ}$ & José & $\mathrm{JxN}$ & Neutro & $\mathrm{MxN}$ \\
\hline & Media & Diff & Media & Diff & Media & Diff & Media & Diff & Media & Diff & Media & Diff \\
\hline \multicolumn{13}{|l|}{ Negociación } \\
\hline Tiempo de envío de propuesta & 1.448 & 0.00111 & 1.447 & -0.00419 & 1.451 & -0.00309 & 3.544 & 0.00131 & 3.543 & -0.00314 & 3.546 & -0.00183 \\
\hline sd & 0.498 & $(0.0484)$ & 0.498 & $(0.0482)$ & 0.499 & $(0.0485)$ & 0.608 & $(0.0461)$ & 0.608 & $(0.0460)$ & 0.604 & $(0.0460)$ \\
\hline Valor promedio de propuestas & 3.810 & -0.167 & 3.977 & 0.0753 & 3.901 & -0.0919 & 3.699 & -0.0710 & 3.770 & 0.0129 & 3.757 & -0.0581 \\
\hline sd & 1.265 & $(0.124)$ & 1.291 & $(0.125)$ & 1.290 & $(0.124)$ & 1.353 & $(0.100)$ & 1.299 & $(0.102)$ & 1.381 & $(0.104)$ \\
\hline Valor promedio de proyectos & 3.971 & -0.0518 & 4.023 & 0.0467 & 3.977 & -0.00510 & 3.874 & 0.0492 & 3.825 & -0.0452 & 3.870 & 0.00398 \\
\hline sd & 0.885 & $(0.0863)$ & 0.894 & $(0.0913)$ & 0.993 & $(0.0915)$ & 0.878 & $(0.0683)$ & 0.924 & $(0.0701)$ & 0.922 & $(0.0683)$ \\
\hline Total de propuestas enviadas & 2.100 & 0.0628 & 2.037 & -0.136 & 2.174 & -0.0737 & 2.181 & 0.0368 & 2.144 & 0.0743 & 2.069 & 0.111 \\
\hline sd & 1.130 & $(0.104)$ & 1.004 & $(0.105)$ & 1.171 & $(0.112)$ & 1.039 & $(0.0789)$ & 1.045 & $(0.0792)$ & 1.042 & $(0.0789)$ \\
\hline Fecha de registro del freelancer & 1.510 & 0.00720 & 1.502 & -0.108 & 1.610 & -0.101 & 1.613 & 0.0672 & 1.546 & 0.00262 & 1.543 & 0.0698 \\
\hline sd & 0.796 & $(0.0787)$ & 0.825 & $(0.0795)$ & 0.820 & $(0.0786)$ & 0.895 & $(0.0652)$ & 0.825 & $(0.0635)$ & 0.848 & $(0.0661)$ \\
\hline \multicolumn{13}{|l|}{ Sin negociación } \\
\hline Tiempo de envío de propuesta & 1.176 & -0.00125 & 1.177 & 0.00125 & 1.176 & 0 & 2.711 & 0 & 2.711 & -0.00553 & 2.717 & -0.00553 \\
\hline sd & 0.382 & $(0.0455)$ & 0.383 & $(0.0455)$ & 0.382 & $(0.0454)$ & 0.643 & $(0.0597)$ & 0.643 & $(0.0599)$ & 0.648 & $(0.0599)$ \\
\hline Valor promedio de propuestas & 3.908 & 0.0787 & 3.830 & -0.198 & 4.028 & -0.120 & 3.871 & 0.0647 & 3.806 & 0.0249 & 3.781 & 0.0896 \\
\hline sd & 1.282 & $(0.148)$ & 1.201 & $(0.141)$ & 1.173 & $(0.146)$ & 1.231 & $(0.118)$ & 1.300 & $(0.121)$ & 1.319 & $(0.118)$ \\
\hline Valor promedio de proyectos & 4.000 & -0.0213 & 4.021 & 0.000150 & 4.021 & -0.0211 & 3.845 & -0.00431 & 3.849 & -0.00494 & 3.854 & -0.00925 \\
\hline sd & 0.907 & $(0.104)$ & 0.841 & $(0.106)$ & 0.934 & $(0.109)$ & 0.898 & $(0.0811)$ & 0.847 & $(0.0814)$ & 0.907 & $(0.0837)$ \\
\hline Total de propuestas enviadas & 2.077 & -0.114 & 2.191 & $0.283^{* *}$ & 1.908 & 0.169 & 2.155 & 0.0905 & 2.065 & $-0.163^{*}$ & 2.227 & -0.0723 \\
\hline sd & 1.130 & $(0.131)$ & 1.068 & $(0.124)$ & 1.024 & $(0.128)$ & 1.086 & $(0.0974)$ & 1.011 & $(0.0959)$ & 1.056 & $(0.0994)$ \\
\hline Fecha de registro del freelancer & 1.641 & 0.0877 & 1.553 & -0.0384 & 1.592 & 0.0493 & 1.638 & 0.0345 & 1.603 & -0.0275 & 1.631 & 0.00703 \\
\hline sd & 0.917 & $(0.106)$ & 0.857 & $(0.101)$ & 0.843 & $(0.105)$ & 0.877 & $(0.0825)$ & 0.901 & $(0.0827)$ & 0.881 & $(0.0815)$ \\
\hline
\end{tabular}

Nota: Las covariables se encuentran transformadas en categóricas por la gran presencia de valores extremos y de missings en la base de línea. El tiempo de envío de la propuesta es una variable que divide el da y medio en que las enviamos en cuatro (mañana, medioda, tarde, noche). Las variables valor promedio de propuestas y proyectos tambien son categóricas (bajo, medio, alto, missing), al igual que el total de propuestas enviadas. La fecha de registro del freelancer es el año de ingreso a la plataforma. 
Figura 6: Plataforma en línea

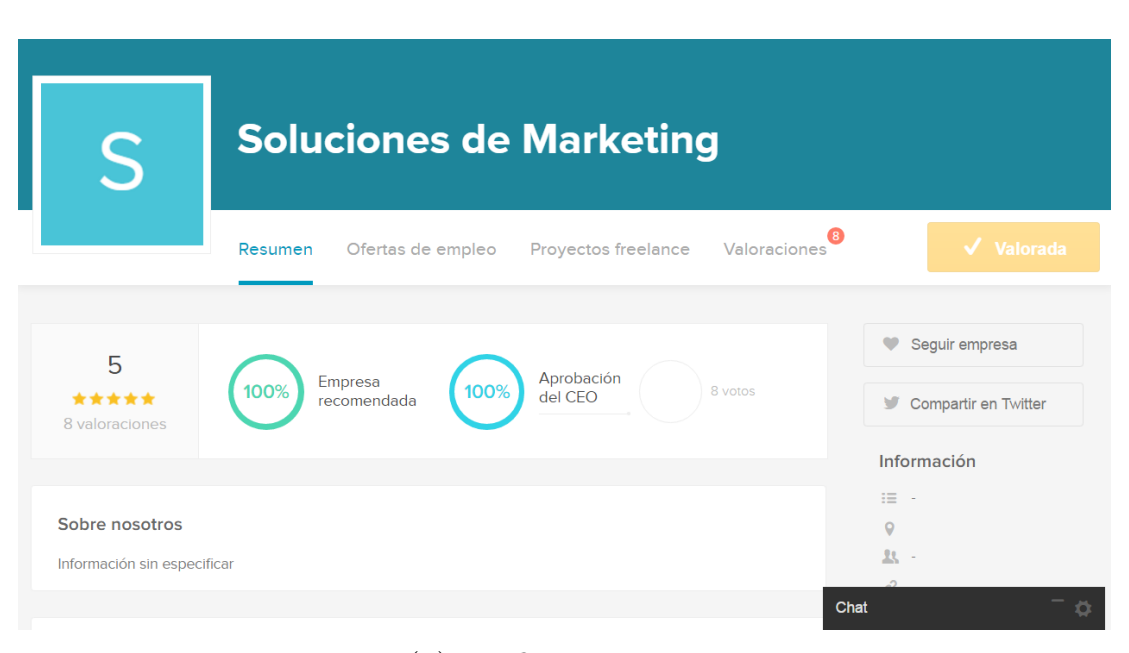

(a) Perfil de empresa
Transcripción simple (precio negociable)

Detalles del trabajo

- Publicación: hace 2 dias y 8 horas

(1) Tiempo restante: 27 dias

7 Tipo de trabajo: proyecto freelance

(3) Ubicación: en cualquier parte del mundo

\section{(3) Presupuesto: sin especificar}

Il Experiencia requerida: Junior (<3 años)

Fecha estimada: 14/07/2016

\section{Descripción del proyecto}

Hola, mi nombre es Maria Carmen García y trabajo en Soluciones de Marketing, una empresa especializada en marketing digital. Estamos buscando una persona para transcribir el video de una ponencia (el texto luego será editado por la empresa para pubilicarse en un blogg). El video trata sobre un tema de marketing y tiene una duracion de 53 minutos, está en idioma españoly l la calidad del audio es muy buena. El plazo de entrega es de hasta 4

Ofrecemos un precio negociable entre 25 y 250 euros por todo el trabajo. El día martes 28 de junio evaluaremos todas las propuestas recibidas. No se contemplarán las propuestas sin el precio total. Por favor, enviame tu propuest lo antes posible. Gracias.

(b) Anuncio empleadora María

Transcripción simple (precio no negociable)

- Proyecto freelance - Termina: en 1 semana

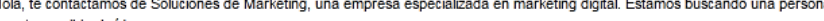

Transcripción simple (precio no negociable)

O Proyecto freelance - Termina: en 1 semana

Hola, mi nombre es Maria Carmen Garciay trabajio en Soluciones de Marketing, una empresa especializada en marketing digttol

Estamos buscando una pers.

Transcripción simple (precio no negociable)

- Proyecto freelance - Termina: en 1 semana

heting, una empresa especializada en marketing digital

Transcripción simple (precio negociable)

O Proyecto freelance - Termina: en 1 semana

Hola, te contactamos de Soluciones de Marketing, una empresa espectializada en marketing digital. Estamos buscando una persona

para transcribir el vide

Transcripción simple (precio negociable)

- Proyecto freelance - Termina: en 1 semana

Hola, mi nombre es Maria Carmen Garcia y y trabajo en Soluciones de Marketing, una empresa especializada en marketing digital

Estamos buscando una pers.

Transcripción simple (precio negociable)

- Proyecto freelance - Termina: en 1 semana

Hola, mi nombre es José Antonio Garcia y trabajo en Soluciones de Marketing, una empresa especializada en marketing digital

stamos buscando una pers.

(c) Listado de anuncios de empleo 